\title{
Amaranthaceae (Caryophyllales) richness and distribution in the state of Aguascalientes, Mexico
}

\section{creative commons}

Botanical Sciences

95 (2): 203-220, 2017

DOI: 10.17129/botsci.909

Copyright: (C) 2017 Sandoval-Ortega et al. This is an open access article distributed under the terms of the Creative Commons Attribution License, which permits unrestricted use, distribution, and reproduction in any medium, provided the original author and source are credited.

Author Contributions:

Manuel Higinio Sandoval-Ortega: Made collections, identify species, drawn the maps, and wrote the main manuscript. María Elena Siqueiros-Delgado: Advised the reserch and species identification. Joaquín Sosa-Ramírez: Advised the reserch and mapping.

Rosa Cerros-Tlatilpa: Advised the reserch and species identification.

\footnotetext{
${ }^{1}$ Herbario HUAA, Departamento de Biología, Centro de Ciencias Básicas, Universidad Autónoma de Aguascalientes, Aguascalientes, Aguascalientes, México.

${ }^{2}$ Centro de Ciencias Agropecuarias, Universidad Autónoma de Aguascalientes, Aguascalientes, Aguascalientes, México.

${ }^{3}$ Lab. Sistemática y Morfología, Facultad de Ciencias Biológicas, Universidad Autónoma del Estado de Morelos, Cuernavaca, Morelos, México.

* Corresponding author: masiquei@correo.uaa.mx
}

\author{
Manuel Higinio Sandoval-Ortega ${ }^{1}$, María Elena Siqueiros-Delgado ${ }^{1,}$, \\ Joaquín Sosa-Ramírez ${ }^{2}$ y Rosa Cerros-Tlatilpa ${ }^{3}$
}

\section{Abstract}

Background: The Amaranthaceae family has a cosmopolitan geographic distribution, with 174 genera and about 2,050 to 2,500 species is the most diverse family within the order Caryophyllales, and includes about $50 \%$ of species of eudicotiledóneas with $\mathrm{C}_{4}$ photosynthesis. Its species live mainly in arid environments, saline habitats and areas of disturbance, many are common weeds.

Questions: How many species of Amaranthaceae family are present in the state of Aguascalientes and where distributed?

Species studied: Species of Amaranthaceae family present in the state of Aguascalientes.

Study site and dates: Aguascalientes, Mexico. From September 2012 to August 2014.

Methodology: Specimens were collected in the field and were identified using especialized literature. Material was revised in various national herbaria. distribution maps were drawn.

Results: In the study area this family is represented by 11 genera and 29 species, eight of these are new records for the state of Aguascalientes and one for Mexico, eighteen are reported as weeds and six are introduced species and naturalized in the state. The genera with more species are Amaranthus and Iresine, with five and six species respectively.

Conclusions: This work expands our understanding of plant resources present in Aguascalientes, as well as the distribution and wealth of the Amaranthaceae family in Mexico.

Key words: Aguascalientes, Amaranthaceae.

\section{Riqueza y Distribución de la familia Amaranthaceae en el Estado de Aguascalien- tes, México \\ Resumen}

Antecedentes: La familia Amaranthaceae tiene una distribución geográfica cosmopolita, con 174 géneros y alrededor de 2,050 a 2,500 especies y es la familia más diversa dentro del orden Caryophyllales, además de incluir cerca del $50 \%$ de especies de eudicotiledóneas con fotosíntesis $\mathrm{C}_{4}$. Sus especies habitan principalmente en ambientes áridos, hábitats salinos y áreas con disturbio, muchas son malezas comunes.

Preguntas: ¿Cuántas especies de la familia Amaranthaceae están presentes en el estado de Aguascalientes y dónde se distribuyen?

Especies estudiadas: Especies de la familia Amaranthaceae presentes en el estado de Aguascalientes.

Sitio de estudio y fechas: Aguascalientes, México. De septiembre del 2012 a agosto del 2014.

Metodología: Se colectaron ejemplares en campo y se identificaron utilizando bibliografía especializada. Se revisó material en diferentes herbarios nacionales. Se elaboraron mapas de distribución.

Resultados: En el área de estudio esta familia está representada por 11 géneros y 29 especies, ocho de éstas son nuevos registros para el estado de Aguascalientes y una para México, dieciocho son reportadas como malezas y seis son especies introducidas y naturalizadas en la entidad. Los géneros con mayor número de especies son Amaranthus e Iresine, con cinco y seis especies respectivamente.

Conclusiones: El presente trabajo amplía el conocimiento de los recursos vegetales presentes en Aguascalientes, así como la riqueza y distribución de la familia Amaranthaceae en México.

Palabras clave: Aguascalientes, Amaranthaceae. 
Figure 1. Municipalities of the state of Aguascalientes. (1) Aguascalientes; (2) Asientos; (3) Calvillo; (4) Cosío; (5) El Llano; (6) Jesús María; (7) Pabellón de Arteaga; (8) Rincón de Romos; (9) San Francisco de los Romo; (10) San José de Gracia; (11) Tepezalá. he state of Aguascalientes is located in the northern center of Mexico, with an area of 5,680 km², it represents approximately the $0.3 \%$ of the total national surface (CONABIO 2008) and consist of 11 municipalities (Figure 1).

Floristically, the state of Aguascalientes belongs to two regions: the Mexican Xerophytic and, to a lesser extent, the Mesoamerican Mountain (Rzedowski 1978). The vegetation of the state of Aguascalientes is incorporated in the NW region by temperate forests of oak, conifer forests and mixed forests, as well as temperate scrub and grasslands; in the SW by a small region with tropical deciduous forest and subtropical scrub, while the central plains of the state are dominated by xeric scrub and grasslands (Rodríguez-Ávalos 2014). Flora represented in each type

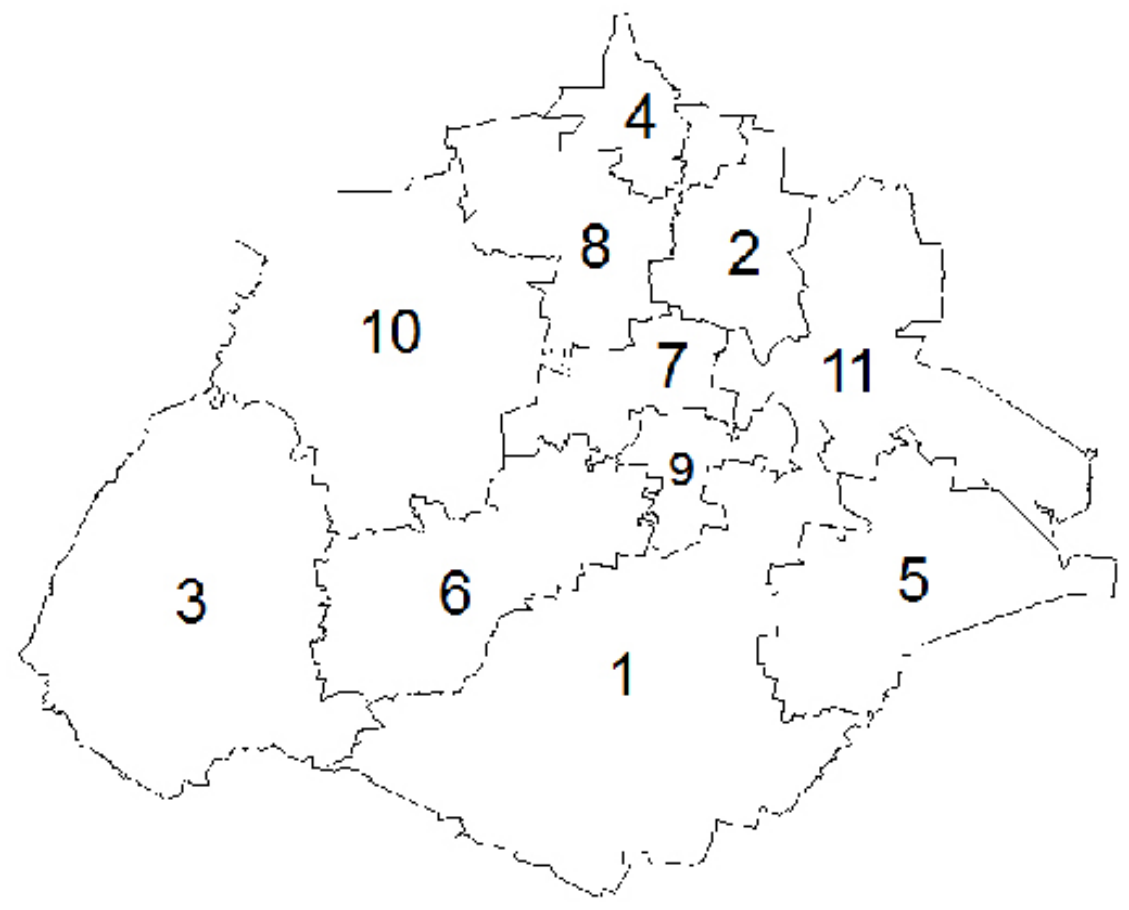

of vegetation is diverse, and its study have a considerable advance (CONABIO 2008, De la Cerda-Lemus 1982, 1985, 1996, 1999, 2000, 2002, 2011, De la Cerda-Lemus et al. 2004, De la Cerda-Lemus \& Herrera-Arrieta 1995, Siqueiros et al. 2011, Siqueiros-Delgado \& GonzálezAdame 2007). However, there are still plant families that have to be reviewed to determine the number of species present and the situation of their populations in the state, as their abundance and distribution. Among them is the family Amaranthaceae, which is very important from the point of view of food and culture, both locally and nationally.

Globally, the family Amaranthaceae has a cosmopolitan geographical distribution, consist of 174 genera and about 2,050 to 2,500 species, being the most diverse family within the order Caryophyllales, and includes about $50 \%$ of eudicotyledon species with $\mathrm{C}_{4}$ photosynthesis (Kadereit et al.2003). Its species live mainly in arid environments, saline habitats and disturbance areas, in addition, many are considered common weeds. It is a family of ecological and economic importance, since it includes several species grown for human consumption, such as amaranth (Amaranthus cruentus L. and A. hypochondriacus L.), quihuicha (A. caudatus L.), quinoa (Chenopodium quinoa Willd.), beets (Beta vulgaris L.), among others. The most important genera in terms of number of species worldwide are: Atriplex L. with 250, Gomphrena L. with 120, Alternanthera Forssk. with 100 and Chenopodium L. with 100 (APG 2009). Before the present study, nine genera and 16 species of this family were reported to Aguascalientes, including those previously considered within the family Chenopodiaceae (García-Regalado et al. 1999).

The aim of this paper is to contribute to the knowledge of the Amaranthaceae family in Mexico, revealing the richness and species distribution of this plant family in the state of Aguascalientes. 


\section{Materials and methods}

Collections were conducted from September 2012 to August 2014, in all municipalities and types of vegetation in the state of Aguascalientes, following the methodology proposed by Lot \& Chiang (1986). In each collection site geographical coordinates based on WGS 84 Datum were taken, and type of vegetation according to Rodríguez-Ávalos (2014) was recorded. The collected material was identified through specialized taxonomic keys (Calderón de Rzedowski 2005 Robertson \& Clemants 2004, Sanchez-Del Pino et al. 2013, Standley 1917, Welsh et al. 2003; Zumaya-Mendoza 2008) and cross checked in the Herbarium of the Autonomous University of Aguascalientes (HUAA). In addition, specimens in the herbarium of the National Institute of Ecology A. C. (IEB) and the National Herbarium of Mexico (MEXU) were reviewed. Distribution maps were made using ArcGis10 software, based on field data and herbarium specimens.

\section{Results}

A total of 387 speciemens were collected in 195 points of the state (Figure 2), which were deposited in the Herbarium of the Autonomous University of Aguascalientes (HUAA).

Figure 2. Collection points.

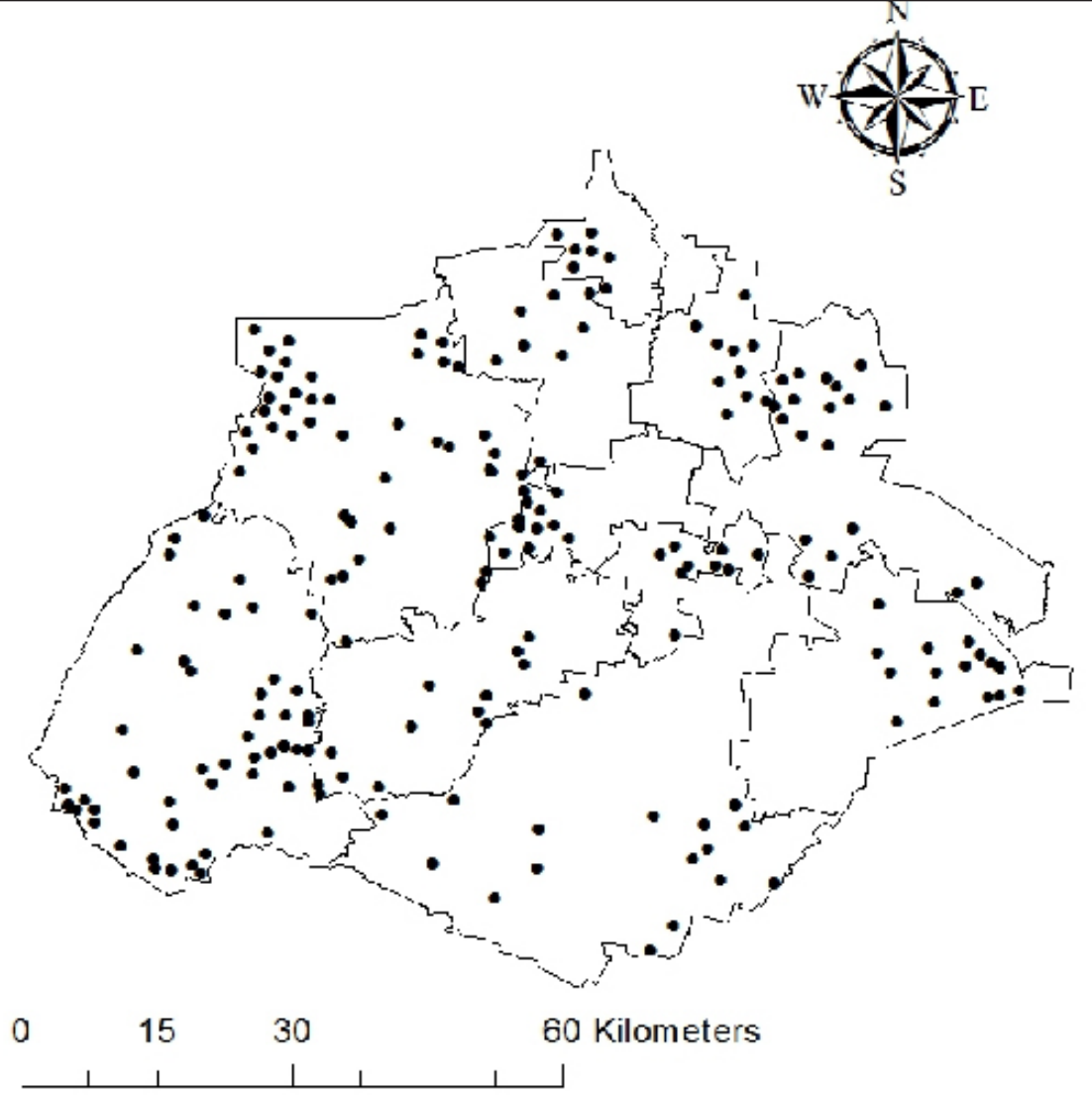

In Aguascalientes, the Amaranthaceae family is represented by 11 genera and 29 species, of which eight were found to be new records for the state (Table 1), according to the literature (De la Cerda-Lemus 1985, Garcia et al. 1999). The genera with more species are: Iresine P. Browne, followed by Amaranthus L., with six and five species, respectively. Six taxa are part of the 23 introduced species reported by Villaseñor-Rios \& Espinosa-García (2004) for the family Amaranthaceae in Mexico (Table 1). In addition, 18 species, representing just over $64 \%$ of the total found, are considered weeds according to Villaseñor-Ríos \& Espinosa-García (1998) and De la Cerda-Lemus (2002), and three of them are considered within of the most important weeds in the world (Holm et al. 1977). The municipality with the highest number of species is Calvillo with 23, and the one with the lowest number is Tepezalá, with just 10 (Figure 3). In 
Table 1. Genera and species of the family Amaranthaceae in Aguascalientes.

\begin{tabular}{|c|c|c|c|}
\hline Genera & Species & $\begin{array}{l}\text { Elevation } \\
\text { (MASL) }\end{array}$ & Vegetation Type \\
\hline Alternanthera & A. caracasana Kunth & $1500-2800$ & $\begin{array}{l}\text { CF, DG, GF, MF, QF, SS, } \\
\text { SV, TF, TG, TS, XS. }\end{array}$ \\
\hline Amaranthus & $\begin{array}{l}\text { A. cruentus } \mathrm{L} \text {. } \\
\text { A. hybridus } \mathrm{L} \text {. } \\
\text { A. palmeri S. Watson } \\
\text { A. polygonoides } \mathrm{L} \text {. } \\
\text { A. powellii S. Watson * } \\
\text { A. spinosus L. * } \\
\text { A. viridis L. * }\end{array}$ & $\begin{array}{l}1800-2200 \\
1500-2800 \\
1500-2800 \\
1600-2800 \\
1700-2300 \\
1500 \\
1800\end{array}$ & $\begin{array}{l}\text { SV. } \\
\text { CF, DG, GF, QF, SS, TF, } \\
\text { TG, TS, SV, XS. } \\
\text { DG, GF, QF, SS, TF, TG, } \\
\text { TS, SV, XS. } \\
\text { GF, QF, SS, SV, XS. } \\
\text { DG, GF, QF, SV, TF, TG, } \\
\text { TS, XS. } \\
\text { SV. } \\
\text { SV. }\end{array}$ \\
\hline Atriplex & $\begin{array}{l}\text { A. muricata Humb. \& Bonpl. ex Willd. } \\
\text { A. semibaccata R. Br. * } \\
\text { A. suberecta Verd. }\end{array}$ & $\begin{array}{l}1900-2100 \\
2000-2200 \\
1700-2000\end{array}$ & $\begin{array}{l}\text { DG, SV, XS. } \\
\text { SV. } \\
\text { DG, SV, XS. }\end{array}$ \\
\hline Chenopodium & $\begin{array}{l}\text { C. album L. } \\
\text { C. berlandieri Moq. } \\
\text { C. fremontii S. Watson }\end{array}$ & $\begin{array}{r}1800-2500 \\
1700-2100 \\
1700-2200\end{array}$ & $\begin{array}{l}\text { DG, GF, MF, QF, SV, TF, } \\
\text { TG, TS, XS. } \\
\text { DG, GF, QF, SS, SV, } \\
\text { TF, XS. } \\
\text { DG, QF, SV, XS. }\end{array}$ \\
\hline Chenopodiastrum & C. murale (L.) S. Fuentes, Uotila \& Borsh & $1700-2200$ & DG, GF, SS, SV, XS. \\
\hline Dysphania & $\begin{array}{l}\text { D. ambrosioides (L.) Mosyakin \& Clemants } \\
\text { D. carinata (R. Br.) Mosyakin \& Clemants * } \\
\text { D. graveolens (Willd.) Mosyakin } \\
\text { \& Clemants }\end{array}$ & $\begin{array}{l}1700-2300 \\
2000-2100 \\
1900-2600\end{array}$ & $\begin{array}{l}\text { DG, GF, SS, SV, XS. } \\
\text { SV, XS. } \\
\text { CF, DG, GF, MF, QF, } \\
\text { SS, SV, TF, TG, XS. }\end{array}$ \\
\hline Froelichia & F. interrupta (L.) Moq. & $1800-2400$ & DG, QF, SS, SV, TG. \\
\hline Gomphrena & G. serrata L. & $1500-2800$ & $\begin{array}{l}\text { DG, GF, MF, QF, SS, SV, } \\
\text { TF, TG, TS, XS. }\end{array}$ \\
\hline Guilleminea & $\begin{array}{l}\text { G. densa (Humb. \& Bonpl. ex } \\
\text { Schult.) Moq. }\end{array}$ & $1500-2500$ & $\begin{array}{l}\text { CF, DG, GF, MF, QF, } \\
\text { SS, SV, TF, TG, TS, XS. }\end{array}$ \\
\hline Iresine & $\begin{array}{l}\text { I. diffusa Humb. \& Bonpl. ex Willd. * } \\
\text { I. grandis Standl. * } \\
\text { I. hartmanii Uline * } \\
\text { I. heterophylla Standl. } \\
\text { I. Iatifolia (M. Martens \& Galeotti) } \\
\text { Benth. \& Hook. f. } \\
\text { I. pringlei S. Watson } \\
\text { I. schaffneri S. Watson }\end{array}$ & $\begin{array}{l}1700 \\
2000-2100 \\
1700-1900 \\
1700-2000 \\
1700-2300 \\
1600-1900 \\
1700-2300\end{array}$ & $\begin{array}{l}\text { TF. } \\
\text { QF } \\
\text { SS, TF. } \\
\text { SS. } \\
\text { GF, QF, SS, XS. } \\
\text { SS, TF. } \\
\text { DG, QF, SS, TF, XS. }\end{array}$ \\
\hline Salsola & S. tragus $\mathrm{L}$. & $1700-2400$ & $\begin{array}{l}\text { CF, DG, GF, MF, QF, SS, } \\
\text { SV, TF, TG, TS, XS. }\end{array}$ \\
\hline
\end{tabular}

$\left(^{*}\right)$ New record to Aguascalientes. Vegetation type: Coniferous Forest (CF), Desert Grassland (DG), Gallery Forest (GF), Mixed Forest (MF), Quercus Forest (QF), Subtropical Scrub (SS), Secondary Vegetation (SV), Deciduous Tropical Forest (TF), Temperate Grassland (TG), Temperate Scrub (TS), Xeric Scrub (XS).

terms of species number by vegetation type, the highest number is found in secondary vegetation, with a total of 22 , followed by xeric scrub with 19 species, and the type of vegetation with the lowest number is the coniferous forest with only five (Figure 4). The species with wider distribution are Alternanthera caracasana Kunth, Amaranthus palmeri S. Watson, Guilleminea densa (Humb. \& Bonpl. ex Schult.) Moq., Gomphrena serrata L. and Salsola tragus L., which are found throughout state (Figure 5A, D, E), mainly in disturbance areas. Among the species with restricted distribution in Aguascalientes stands out the genus Iresine (Figure 5F), because three species are found only in the municipalities of Calvillo and San José de Gracia. So far, Iresine grandis Standl. has only been located in a preserved area of Sierra del Laurel, Calvillo; to this same municipality is also restricted I. hartmanii Uline and I. diffusa Humb. \& Bonpl. ex 
Figure 3. Number of species by municipalitie. Aguascalientes (AGS), Asientos (ASIE), Calvillo (CAL), Cosío (COS), El Llano (ELL), Jesús María (JM), Pabellón de Arteaga (PA), Rincón de Romos (RR), San Francisco de los Romo (SFR), San José de Gracia (SJG), Tepezalá (TEP).

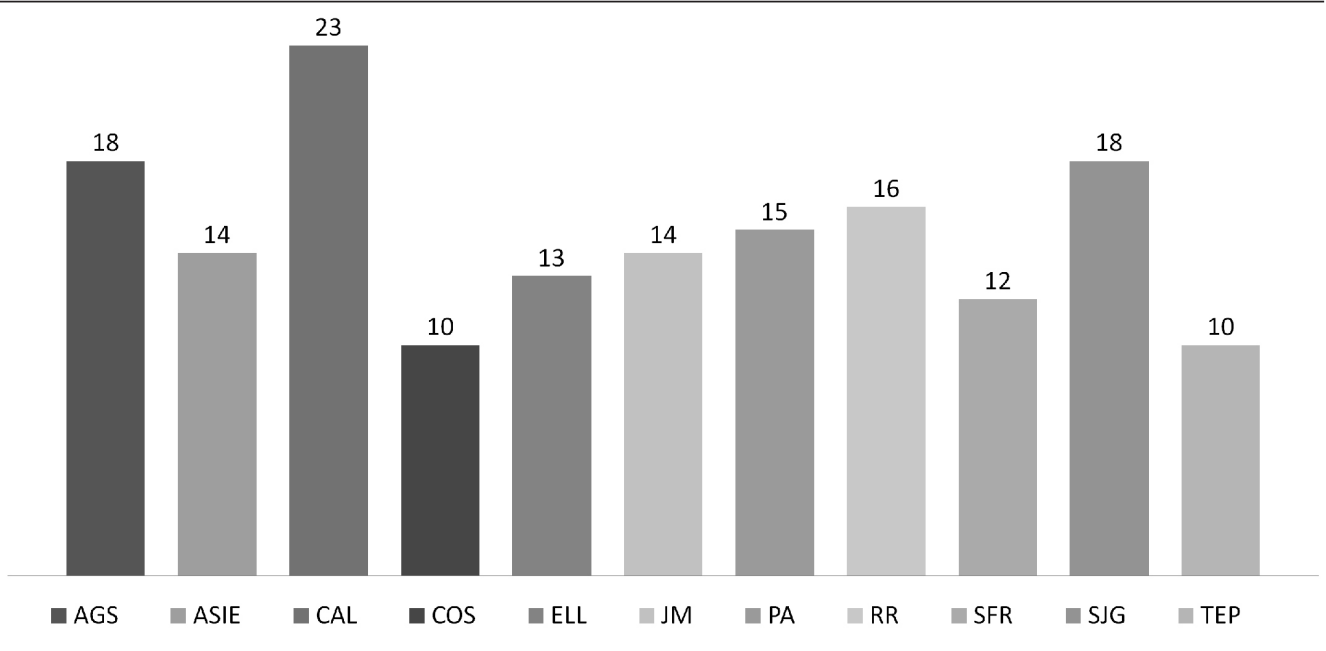

Figure 4. Number of species by vegetation type. Coniferous Forest (CF), Desert Grassland (DG), Gallery Forest (GF), Mixed Forest (MF), Quercus Forest (QF), Subtropical Scrub (SS), Secondary Vegetation (SV), Deciduous Tropical Forest (TF), Temperate Grassland (TG), Temperate Scrub (TS), Xeric Scrub (XS).

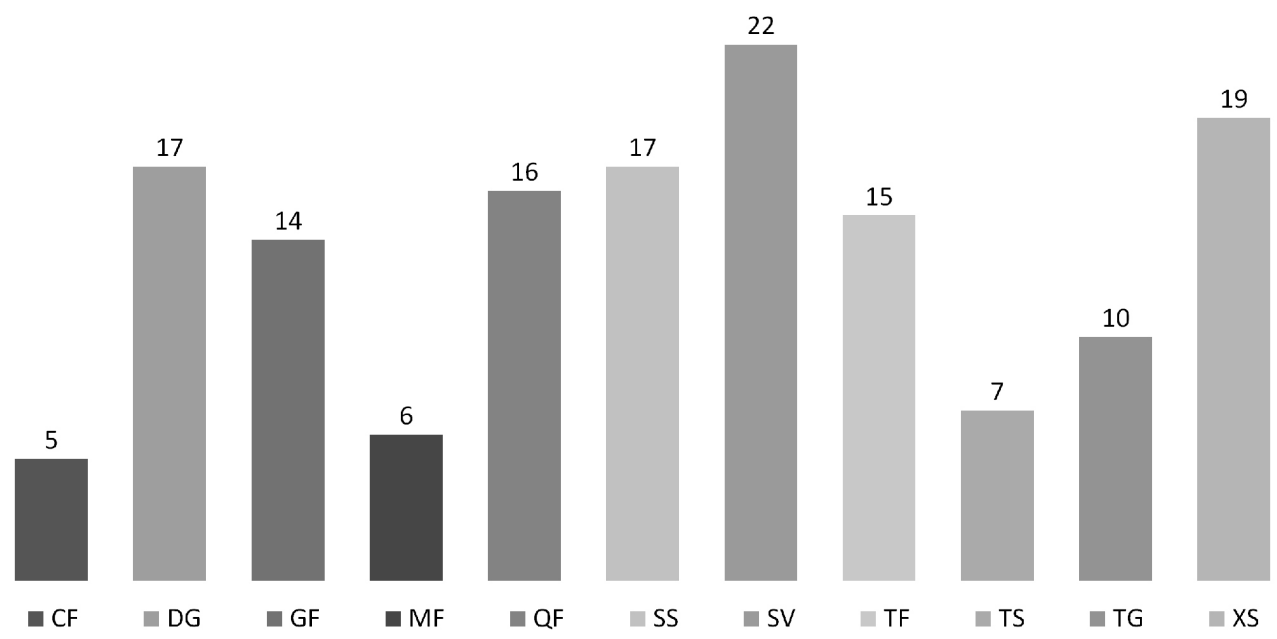

Willd. (Fig 5F.), both in subtropical vegetation; likewise I.pringlei S. Watson is distributed only in the municipalities of Calvillo and San José de Gracia (Figure 5F). Other species of restricted distribution in the state are Dysphania carinata (R. Br.) Mosyakin \& Clemants (Figure 6A) and Atriplex semibaccata R. Br. (Figure 5B, 6B), both introduced and located north and northwest of the state; the first is found in arid and open areas of the municipalities of Cosio, Rincon de Romos and El Llano, and is reported to Mexico for the first time in this work; while the second is restricted to the municipality of Asientos, in lime soils and road sides. Another rare species in the state is Amaranthus spinosus L. (Figure 6C), which is restricted to disturbance areas of the municipality of Calvillo (Figure 5A) at an elevation of 1,500 m.

\section{Discussion}

In Aguascalientes, $41.7 \%$ of the total state area has been transformed with anthropic purposes, and $36.9 \%$ of the surface covered with natural vegetation is considered secondary vegetation, product of grazing or other activities (Rodríguez-Ávalos 2014), that's why some species considered weeds are commonly found in all types of vegetation in the state. As for species that behave like weeds, the family Amaranthaceae is one of the most important in arid areas because they are adapted to desert and semi-desert environments and saline habitats, Kadereit et al. (2003) mentions that many species of the family Amaranthaceae can develop successfully in arid areas, as they have $\mathrm{C}_{4}$ photosynthesis and can use water more efficiently and withstand water stress, allowing them to settle in inaccessible niches for other plants. Most species belonging to this 

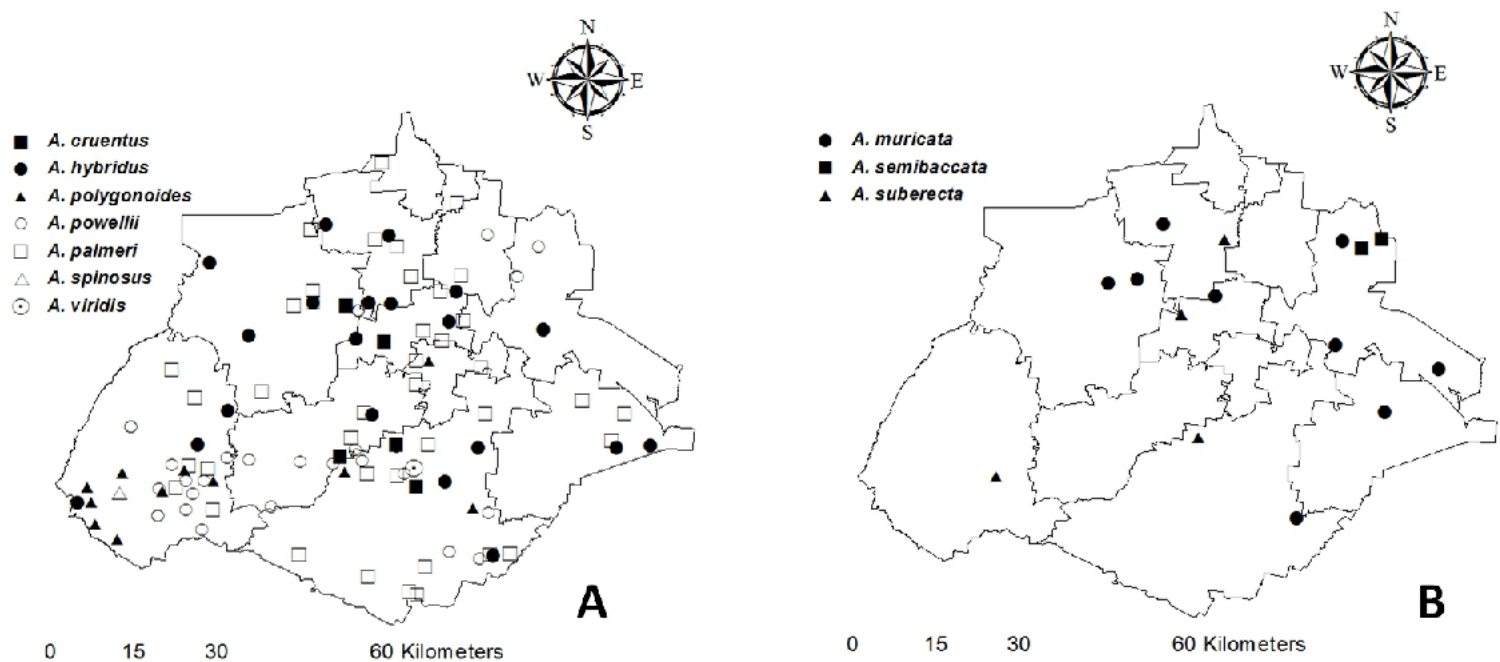

\begin{tabular}{l}
$0 \quad 15 \quad 30 \quad 60$ Kilometers \\
\hline
\end{tabular}
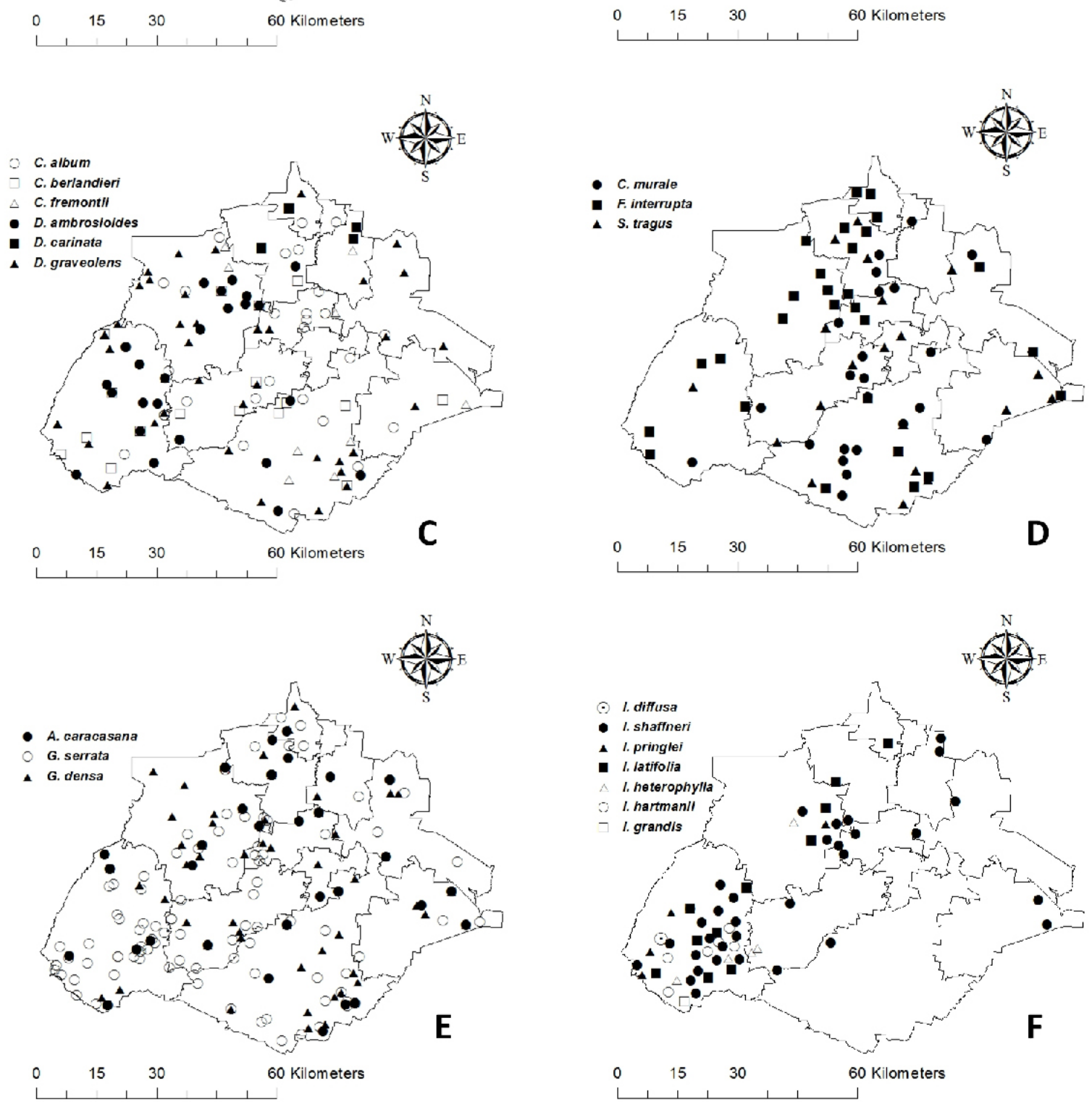

Figure 5. Distribution maps. A) species of the genus Amaranthus; B) species of the genus Atriplex; C) species of the genera Chenopodium and Dysphania; D) Chenopodiastrum murale, Froelichia interrupta and Salsola tragus; E) Alternanthera caracasana, Gomphrena serrata and Guilleminea densa; F) species of the genus Iresine. 


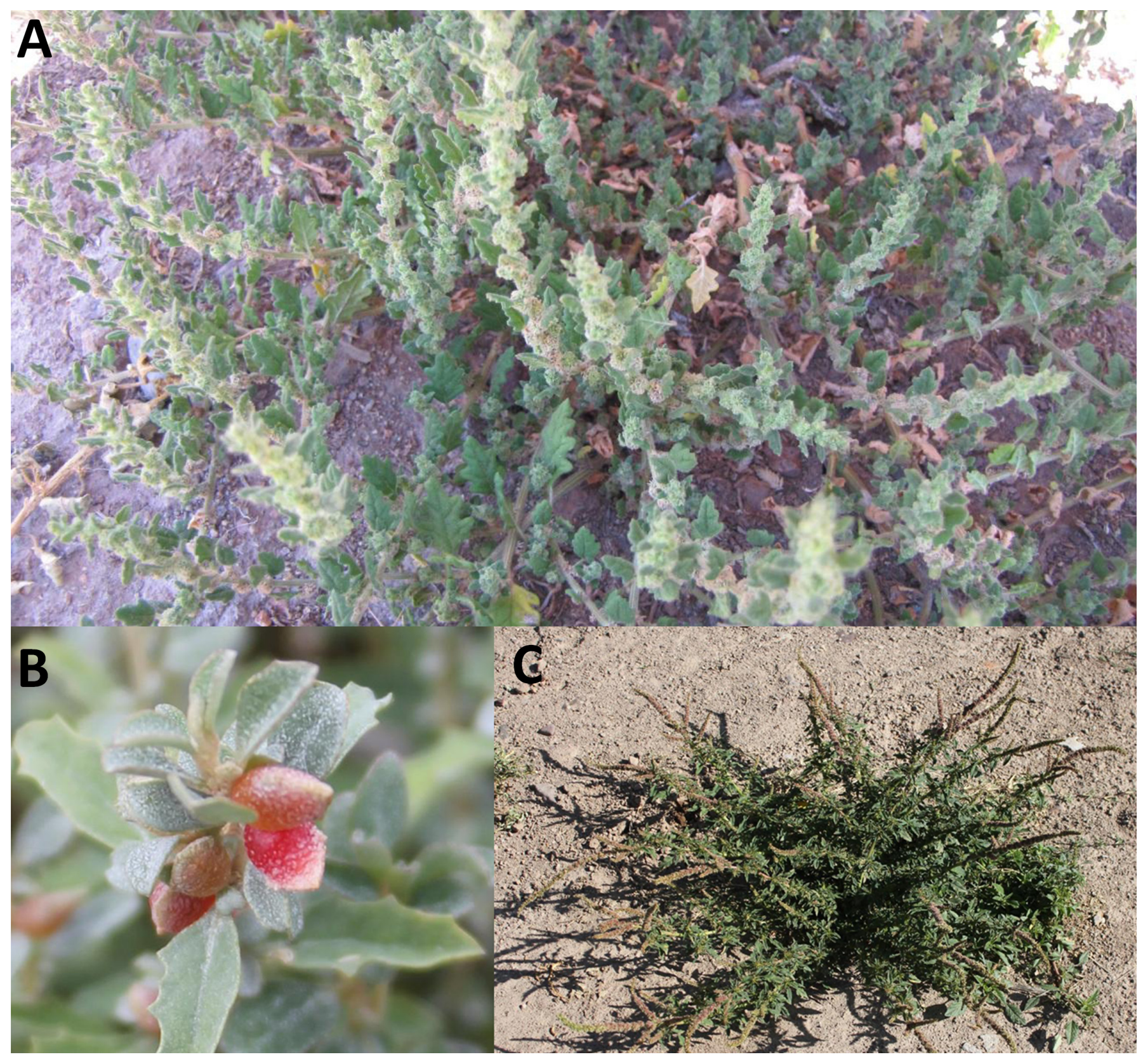

Figure 6. A) Dysphania carinata (R. Br.) Mosyakin \& Clemants. B) Atriplex semibaccata R. Br. C) Amaranthus spinosus L.

family in Aguascalientes are reported as weeds by Villaseñor-Ríos \& Espinosa-García (1998). However, although Amaranthus spinosus (Figure 5C) is considered one of the most common and most widely distributed weeds in tropical areas of the New World (Sauer 1967), is actually an uncommon plant in Aguascalientes.

It is remarkable that within this family exist morphollogicaly similar species that can be confused. Such is the case of Chenopodium album and C. berlandieri, both common weeds and often sympatric in North America (Welsh et al. 2003). The first is a cosmopolitan species native to Eurasia and the second is native and semi-domesticated. Chenopodium berlandieri subsp. nuttalliae Wilson \& Heiser, also known as "Huanzontle", is consumed as a vegetable in Mexico (Jellen et al. 2011). Chenopodium album and C. berlandieri are close related species with wide phenotypic variability (Welsh et al.2003), making it difficult to identify them only with vegetative characters; the main distinguishing feature is fruit morphology, in C. album the 
utricle is smooth, while in C. berlandieri is alveolated. A similar case happens with Amaranthus hybridus and A. powelli, both species of the A. hybridus complex (Costea 2001, Sauer 1950) and morphologically similar besides being sympatric, which tend to be confused in their vegetative state (Sauer 1967), however, they have a number of distinctive morphological characteristics as the trichomes and bracts (Costea et al. 2001, Costea \& Tardif 2003); nevertheless, many individuals with intermediate characteristics were observed, probably the result of hybridization, which according to some authors (Costea et al. 2001, Sauer 1950, Weaver \& McWilliams 1980) is not rare among sympatric species of A. hybridus complex. Sauer (1950) suggests that much of the morphological variation in A. hybridus is due to introgression of other nearby species, and although most hybrids are sterile, fertile populations can be found.

Dysphania carinata (Figure 6A) is a native species to Australia (Clemants \& Mosyakin 2003), currently introduced in Africa, Europe, United States, and South America; for Mexico is reported for the first time in this paper. It is believed that its distribution can be extended to the state of Zacatecas because collections of this species were made close to the northern boundary of the state (Figure 5C).

The Amaranthaceae family is widely distributed in Aguascalientes, its members can be found in all types of vegetation present in the state and none of the species is within the NOM-059SEMARNAT-2010. Species richness of this family in Aguascalientes is comparable with larger states, such as Queretaro, for which 22 species are reported (Argüelles et al. 1991), and Michoacán with about 34 (Rodriguez -Jiménez \& Garduño-Espinosa 1995). However, it is considerably lower compared to states like Baja California, which has 68 species (Wiggins 1980) and Veracruz with 87 (Sosa \& Gómez-Pompa 1994). This work extends the knowledge of plant resources with which account Aguascalientes and distribution and richness of the Amaranthaceae family in Mexico.

\section{Acknowledgement}

We are grateful for the financial support as part of the project JF140 of CONABIO, to CONACYT for the scholarship 354518, and to the herbarium team of the Autonomous University of Aguascalientes (HUAA) for their support during this project.

\section{Literature cited}

The Angiosperm Phylogeny Group. 2009. An Update of the Angiosperm Phylogeny Group classification for the orders and families of flowering plants: APG III. Botanical Journal of the Linnean Society 161: 105-121. DOI: 10.1111/j.1095-8339.2009.00996.x

Argüelles E, Fernández R, Zamudio S. 1991. Listado florístico preliminar del estado de Querétaro. Flora del Bajío y de regiones adyacentes, Fascículo complementario II. Páztcuaro: Instituto de Ecología A.C.

Calderón de Rzedowski G. 2005. Amaranthaceae. In: Calderón de Rzedowski G, Rzedowski J, eds. Flora fanerogámica del Valle de México. D.F.: Comisión Nacional para el Conocimiento y Uso de la Biodiversidad and Instituto de Ecología AC, 124-132.

Clemants SE, Mosyakin SL. 2003. Dysphania . In: Editorial Committee, eds. Flora of North America, Vol. 4. New York and Oxford.

CONABIO. 2008. La Biodiversidad en Aguascalientes: Estudio de Estado. D.F: Comisión Nacional para el Conocimiento y Uso de la Biodiversidad, Instituto del Medio Ambiente del Estado de Aguascalientes and Universidad Autónoma de Aguascalientes.

Costea M, Sanders A, Waines G. 2001. Preliminary results toward a revision of the Amaranthus hybridus species complex (Amaranthaceae). Sida 19: 931-974.

Costea M, Tardif FJ. 2003. The bracteoles in Amaranthus (Amaranthaceae): their morphology, structure, function and taxonomic significance. Sida 20: 969-985.

De la Cerda-Lemus M. 1982. Estudio taxonómico ecológico de la flora y fauna del estado de Aguascalientes. Aguascalientes: Universidad Autónoma de Aguascalientes.

De la Cerda-Lemus M. 1996. Las gramíneas de Aguascalientes. Aguascalientes: Universidad Autónoma de Aguascalientes.

De la Cerda-Lemus M. 1999. Encinos de Aguascalientes. Aguascalientes: Universidad Autónoma de Aguascalientes. 
Received:

June 25th, 2016

Accepted:

October 12th, 2016
De la Cerda-Lemus M. 2000. Álamos y Sauces del Estado de Aguascalientes. Investigación y Ciencia 23: 17-24.

De la Cerda-Lemus ME. 2002. Malezas de Aguascalientes. MSc. Thesis, Universidad Nacional Autónoma de México.

De la Cerda-Lemus M. 2011. La familia Burseraceae en el Estado de Aguascalientes, México. Acta Botanica Mexicana 94: 1-25.

De la Cerda-Lemus M, García-Regalado G, González-Adame G. 2004. Contribución al conocimiento de la Flora del estado de Aguascalientes. Scientiae Naturae 6: 5-7.

De la Cerda-Lemus M, Herrera-Arrieta Y. 1995. New Poaceae distribution records for Aguascalientes, MEXICO Cuarenta y tres nuevos registros de Gramíneas para el estado de Aguascalientes. Madroño 42: 396-405.

De la Cerda-Lemus M. 1985. Avances de la Flora de Aguascalientes. Aguascalientes: Universidad Autónoma de Aguascalientes. Aguascalientes.

García-Regalado G, Rosales-Carrillo O, De La Cerda-Lemus M, Siqueiros-Delgado ME. 1999. Listado Florístico del estado de Aguascalientes. Scientiae Naturae 1: 5-51.

Holm LRG, Plucknett DL, Pancho JV, Herberger JP. 1977. The world's worst weeds. Distribution and biology. Honolulu: University Press of Hawaii.

Jellen NE, Kolano AB, Sederberg CM, Bonifacio A, Maughan JP. 2011. Chenopodium. In: Kole C, ed. Wild Crop Relatives: Genomic and Breeding Resources. Legume Crops and Forages. Berlin-Heidelberg: Springer-Verlag, 60-86.

Kadereit G, Borsch T, Weising K, Freitag H. 2003. Phylogeny of Amaranthaceae and Chenopodiaceae and the evolution of $\mathrm{C}_{4}$ photosynthesis. International Journal of Plant Sciences 164: 959-986. DOI: $10.1086 / 378649$

Lot A, Chiang F. 1986. Manual de herbario. Administración y manejo de colecciones, técnicas de recolección y preparación de ejemplares botánicos. D.F: Consejo Nacional de la Flora de México AC.

Robertson K, Clemants S. 2004. Amaranthaceae. In: Editorial Committee, eds. Flora of North America, Vol. 4. New York and Oxford.

Rodríguez-Ávalos JA. 2014. Distribución geográfica y descripción de las comunidades vegetales naturales de Aguascalientes. PhD Thesis, Universidad Autónoma de Aguascalientes.

Rodríguez Jiménez LS, Espinosa Garduño J. 1995. Listado florístico del estado de Michoacán. Sección I (Gymnospermae; Angiospermae: Acanthaceae-Commelinaceae). Flora del Bajío y de regiones adyacentes, Fascículo complementario VI. Páztcuaro: Instituto de Ecología AC.

Rzedowski J.1978. Vegetación de México. D.F: Limusa.

Sánchez-Del Pino I, Espadas C, Pool R. 2013. Taxonomy and richness of nine genera of Amaranthaceae ss (Caryophyllales) in the Yucatan Peninsula Biotic Province. Phytotaxa 107: 1-74. DOI:10.11646/phytotaxa.107.1.1

Sauer JD. 1950. Amaranths: A survey of their history and classification. Annals of the Missouri Botanical Garden 37: 561-632. DOI: 10.2307/2394403

Sauer JD. 1967. The Grain Amaranths and Their Relatives: A Revised Taxonomic and Geographic Survey. Annals of the Missouri Botanical Garden 54: 103-137. DOI: 10.2307/2394998

Siqueiros-Delgado ME, González-Adame G. 2007. Helechos y plantas afines del estado de Aguascalientes. México, D.F: Comisión Nacional para el Conocimiento y Uso de la Biodiversidad and Universidad Autónoma de Aguascalientes.

Siqueiros-Delgado ME, García-Regalado G, Rosales-Carrillo O, Flores MC. 2011. Malvales del Estado de Aguascalientes: Bombacaceae, Cistaceae, Malvaceae, Sterculiaceae y Tiliaceae. Aguascalientes: Universidad Autónoma de Aguascalientes.

Sosa V, Gómez-Pompa A. 1994. Lista florística. Flora de Veracruz, Fascículo 82.

Standley PC. 1917. Amaranthaceae. North American Flora 21, part 2: 95-169.

Villaseñor-Ríos JL, Espinosa-García FJ. 1998. Catálogo de Malezas de México. México, D.F: Universidad Nacional Autónoma de México. Consejo Nacional Consultivo Fitosanitario, and Fondo de Cultura Económica. México.

Villaseñor JL, Espinosa-García FJ. 2004. The alien flowering plants of Mexico. Diversity and Distributions 10: 113-123. DOI: 10.1111/j.1366-9516.2004.00059.x

Weaver SE, McWilliams EL. 1980. The Biology of Canadian Weeds.: 44. Amaranthus retroflexus L., A. powellii S. Wats. and A. hybridus L. Canadian Journal of Plant Sciences 60: 1215-1234.

Welsh S, Cropton C, Clemants S. 2003. Chenopodiaceae. Flora of North America. New York and Oxford: Oxford University.

Wiggins I. 1980. Flora of Baja California. California: Stanford University Press.

Zumaya-Mendoza SG. 2008. Las especies mexicanas del género Iresine P. Browne (Amaranthaceae). MSc Thesis, Universidad Nacional Autónoma de México. 


\section{EXAMINED SPECIMENS:}

Alternanthera caracasana Kunth

Aguascalientes: lado W de Cd. Universitaria UAA, Rosales-Carrillo 1148 (HUAA); 1 km al SW de José María Morelos (Cañada Honda), Rosales-Carrillo 1242 (HUAA); Entre presa y la Hacienda San Bartolo, Rosales-Carrillo 1308 (HUAA); Rumbo al Cerro de Los Gallos, Rosales-Carrillo 1965 (HUAA); Presa El Niagara, Siqueiros-Delgado 2151 (HUAA). Asientos: Presa El Ilavero,

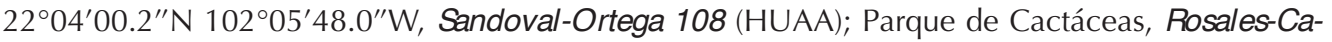
rrillo 1248 (HUAA). Calvillo: $0.5 \mathrm{~km}$ al O de la Barranca El Pilar, $22^{\circ} 04^{\prime} 14.7^{\prime \prime} \mathrm{N} 102^{\circ} 43^{\prime} 47.6^{\prime \prime} \mathrm{W}$, Sandoval-Ortega 135 (HUAA); Los Alisos, Rosales-Carrillo 1304 (HUAA); 4 km al NW del Temazcal, Rosales-Carrillo 1335 (HUAA); Río Gil, Rosales-Carrillo 1365 (HUAA); Río Mal Paso, RosalesCarrillo 1418 (HUAA); Presa Cerro Blanco, Rosales-Carrillo 1860 (HUAA). Cosio: Tanque La Punta, al E del Poblado La punta, Rosales-Carrillo 2051 (HUAA) El Llano: $3 \mathrm{~km}$ al N de Ojo de Agua de Crucitas, Rosales \& Croce 24 (HUAA); $1.3 \mathrm{~km}$ al S de Fco. Sarabia, 21 ${ }^{\circ} 57^{\prime} 22.9^{\prime \prime} \mathrm{N} 102^{\circ} 01^{\prime} 01.1^{\prime \prime} \mathrm{W}$, Sandoval-Ortega 760 (HUAA). Jesús María: San Isidro, carr. Calvillo-Tapias Viejas, De la CerdaLemus 5737 (HUAA). Pabellón de Arteaga: Libramiento a Luis Moya, Frente a Colonia Campestre, Rosales-Carrillo 1467 (HUAA); 1.5 km del entronque Pabellón de Arteaga - Rincón de Romos, De la Cerda-Lemus 5687 (HUAA). Rincón de Romos: Lado W del poblado La Escalera, RosalesCarrillo 1166 (HUAA); Barranca Sierpens al W de Las Camas, Rosales-Carrillo 1217 (HUAA). San Francisco de Los Romo: Puertecito de La Virgen, $21^{\circ} 58^{\prime} 18.9^{\prime \prime} \mathrm{N} 102^{\circ} 14^{\prime} 43.1^{\prime \prime} \mathrm{W}$, Sandoval-Ortega 995 (HUAA). San José de Gracia: $3 \mathrm{~km}$ al NW de la cortina de la presa El Jocoqui, $22^{\circ} 08^{\prime} 04.5^{\prime \prime} \mathrm{N}$ $102^{\circ} 22^{\prime} 55.4^{\prime \prime}$ W, Sandoval-Ortega 307 (HUAA); 2 km al SE de Arroyo Viejo Camino a Potrero de Los López, Rosales-Carrillo 1271 (HUAA); Al Sur de Potrero de Los López, Rosales-Carrillo 1292 (HUAA). Tepezalá: El Carmen, 4.5 km al W de Tepezalá, De la Cerda-Lemus 5964 (HUAA).

\section{Amaranthus cruentus $\mathrm{L}$.}

Aguascalientes: Universidad Autónoma de Aguascalientes, Sandoval-Ortega 957 (HUAA); Baldío Fracc. Las Américas, $21^{\circ} 52^{\prime} 10.9^{\prime \prime} \mathrm{N} 102^{\circ} 18^{\prime} 10.1^{\prime \prime} \mathrm{W}$, Sandoval-Ortega 994 (HUAA). Jesús María:

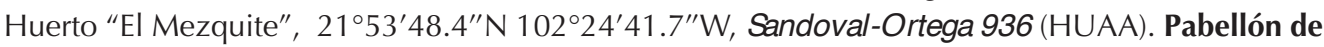
Arteaga: Extremo W del Poblado El Garabato, $22^{\circ} 05^{\prime} 04.8^{\prime \prime} \mathrm{N} 102^{\circ} 20^{\prime} 26.2^{\prime \prime} \mathrm{W}$, Sandoval-Ortega 997 (HUAA). San José de Gracia: $1 \mathrm{~km}$ al SE del Municipio San José de Gracia, 22 $08^{\prime} 31.4^{\prime \prime} \mathrm{N}$ $102^{\circ} 24^{\prime} 06.0^{\prime \prime} \mathrm{N}$, Sandoval-Ortega 998 (HUAA).

\section{Amaranthus hybridus L.}

Aguascalientes: $3 \mathrm{~km}$ al E de El Cuervo, $21^{\circ} 43^{\prime} 38.6^{\prime \prime} \mathrm{N} 102^{\circ} 07^{\prime} 55.0^{\prime \prime} \mathrm{W}$, Sandoval-Ortega 704 (HUAA); $1.5 \mathrm{~km}$ al E de Aguascalientes, Ojo de Agua de Palmitas, De la Cerda 5870 (HUAA). Asientos: Presa El Ilavero, $22^{\circ} 04^{\prime} 00.2^{\prime \prime} \mathrm{N} 102^{\circ} 05^{\prime} 48.0^{\prime \prime} \mathrm{W}$, Sandoval-Ortega 111 (HUAA); Nuevo Valle, $6 \mathrm{~km}$ al NE de Villa Juárez, De la Cerca-Lemus 5048 (HUAA). Calvilo: Presa Nueva, $1.5 \mathrm{~km}$ al N de Presa de los Serna, $21^{\circ} 49^{\prime} 16.6^{\prime \prime} \mathrm{N} 102^{\circ} 50^{\prime} 21.1^{\prime \prime} \mathrm{W}$, Sandoval-Ortega 851 (HUAA); Cerro La Iguana, $4.5 \mathrm{~km}$ al NNW de El Sauz, $21^{\circ} 54^{\prime} 56.6^{\prime \prime} \mathrm{N} 102^{\circ} 38^{\prime} 36.8^{\prime \prime} \mathrm{W}$, Sandoval-Ortega 897a(HUAA). El Llano: $1.7 \mathrm{~km}$ al NE de El Tule, $21^{\circ} 54^{\prime} 45.4^{\prime \prime} \mathrm{N} 101^{\circ} 55^{\prime} 06.4^{\prime \prime} \mathrm{W}$, Sandoval-Ortega 439 (HUAA); Arroyo el aguacero, a $2.5 \mathrm{~km}$ al NE de El Tule, $21^{\circ} 54^{\prime} 50.0^{\prime \prime} \mathrm{N} 101^{\circ} 54^{\prime} 25.7^{\prime \prime} \mathrm{W}$, Sandoval-Ortega

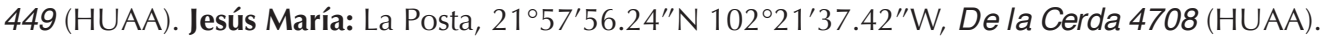
Pabellón de Arteaga: Norte de Ojo Zarco, carr. Asientos, De la Cerda 5942 (HUAA). Rincón de Romos: Las Camas, 22 $2^{\circ} 6^{\prime} 30.9^{\prime \prime} \mathrm{N} 102^{\circ} 26^{\prime} 05.3^{\prime \prime} \mathrm{W}$, Arroyo-Chavez 221 (HUAA); $11.75 \mathrm{~km}$ al NW de Rincón de Romos, $22^{\circ} 08^{\prime} 49.8^{\prime \prime} N 102^{\circ} 21^{\prime} 52.9^{\prime \prime}$ W, Sandoval-Ortega 301 (HUAA); Lado W del poblado La Escalera. Rosales-Carrillo 1170 (HUAA), El Milagro, De la Cerda 4778 (HUAA). San Francisco de Los Romo: a $5 \mathrm{~km}$ al W de Hacienda el Garabato, $22^{\circ} 05^{\prime} 17.7^{\prime \prime} \mathrm{N} 102^{\circ} 23^{\prime} 08.8^{\prime \prime} \mathrm{W}$, Sandoval-Ortega $835 a(\mathrm{HUAA}) ;$ a $4.7 \mathrm{~km}$ al W de Hacienda el Garabato, $22^{\circ} 04^{\prime} 57.8^{\prime \prime} \mathrm{N} 102^{\circ} 23^{\prime} 0.8^{\prime \prime} \mathrm{W}$, Sandoval-Ortega 829 (HUAA). San José de Gracia: La Cienega, Sierra San Blas de Pabellón, Rosales-Carrillo 1118 (HUAA); Mesa Montoro, Rosales-Carrillo 2121 (HUAA); Estación Biológica Agua Zarca, Rosales-Carrillo 1585 (HUAA); 6.8 km al NNE de San Antonio de Los Ríos, De la CerdaLemus 5138 (HUAA). 
EXAMINED SPECIMENS: Continuation.

Amaranthus palmeri S. Watson

Aguascalientes: $1 \mathrm{~km}$ al SE de Tanque de Los Jimenez, $21^{\circ} 42^{\prime} 03.1^{\prime \prime} \mathrm{N} 102^{\circ} 21^{\prime} 57.7^{\prime \prime} \mathrm{W}$, SandovalOrtega 780 (HUAA); 3 km al E de El Cuervo, 21 $43^{\prime} 38.6^{\prime \prime} \mathrm{N} 102^{\circ} 07^{\prime} 55.0^{\prime \prime} \mathrm{W}$, Sandoval-Ortega 705 (HUAA); 200 m del entronque carr. Aguascalientes-Encarnación de Díaz, Cerro Los Gallos, De la Cerda-Lemus 5805 (MEXU; HUAA); Viñedos San Marcos, carr. Aguascalientes-Zacatecas, De la Cerda-Lemus 4973 (HUAA); Granja San Gabriel, $3 \mathrm{~km}$ al S del Crucero Calvillo-Villa Hidalgo, De la Cerda-Lemus 4801 (HUAA); lado W de Cd. Universitaria UAA, Rosales-Carrillo 1150 (HUAA); 1 km al S del Ejido Peñuelas, Rosales \& Croce 133 (HUAA); Entre presa y la Hacienda San Bartolo, Rosales-Carrillo 1317 (HUAA). Calvillo: Cruce Camino Río Gil - El Sauz, 2151'29.5”N 102 37'59.4"W, Sandoval-Ortega 113 (HUAA); $500 \mathrm{~m}$ al SW de El Temazcal, $21^{\circ} 50^{\prime} 44.3^{\prime \prime} \mathrm{N}$ $102^{\circ} 40^{\prime} 43.6^{\prime \prime} \mathrm{W}$, Sandoval-Ortega 167 (HUAA); Barranca La Botita, 2150'13.2”N 102³9'02.6”'W,

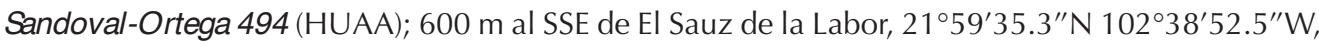
Sandoval-Ortega 740 (HUAA); Arroyo al S de Palo Alto, Rosales-Carrillo 1387 (HUAA); Río Gil, Rosales-Carrillo 1364 (HUAA). Cosío: $1.3 \mathrm{~km}$ al W del Salero, Rosales \& Croce 97 (HUAA). El Llano: 1 km al SW de la Luz, De la Cerda-Lemus 5528 (HUAA); Carr. Palo Alto, Frente a Sabro Pollo, Rosales \& Croce 28 (HUAA); Ojo de Agua de Crucitas, Rosales \& Croce 4 (HUAA). Jesús María: La Posta, De la Cerda-Lemus 4732 (HUAA); Cortina de la Presa El Jarabe a $4.5 \mathrm{~km}$ al SE de

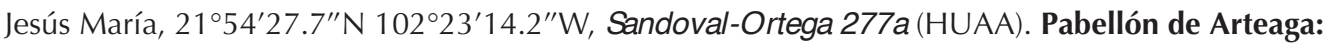
Libramiento a Luis Moya, Frente a Colonia Campestre, Rosales-Carrillo 1472 (HUAA); N de Ojo Zarco, Carretera a Asientos, De la Cerda-Lemus 5938a (HUAA); Faldas del Cerro El Chiquihuite, Rosales \& Croce 213 (HUAA). Rincón de Romos: El Salitrillo, Rosales \& Croce 228 (HUAA); 800 $\mathrm{m}$ al S del entronque Valle de Las Delicias, carr. A Cosío, De la Cerda 4883 (HUAA); Lado W del poblado La Escalera, Rosales-Carrillo 1162 (HUAA); Barranca Sierpens, al W de Las Camas, Rosales-Carrillo 1220 (HUAA). San Francisco de Los Romo: 2.3 km al E de Borrotes, 2202'29.2"N $102^{\circ} 10^{\prime} 57.0^{\prime \prime} \mathrm{W}$, Sandoval-Ortega 681 (HUAA); $3 \mathrm{~km}$ al S de San Francisco de Los Romo, De la Cerda-Lemus 4807 (HUAA); Granja Las Moras, 1.5 km al N del entronque Pabellón- Emiliano Zapata, De la Cerda-Lemus 4817 (HUAA). San José de Gracia: 2 km al SW de San Antonio de Los Ríos, De la Cerda-Lemus 5142 (HUAA); 1 km al NE de San Antonio de los Ríos, De la Cerda-Lemus 5727 (HUAA); Arroyo Viejo al SW de Paredes, Rosales-Carrillo 1258 (HUAA).

\section{Amaranthus polygonoides L.}

Aguascalientes: Cañada al W de la presa El Muerto, Rosales-Carrillo 1846 (HUAA); A $2.5 \mathrm{~km}$ al NW de Calvillito, $21^{\circ} 48^{\prime} 18.6^{\prime \prime} \mathrm{N} 102^{\circ} 10^{\prime} 14.5^{\prime \prime} \mathrm{W}$, Sandoval-Ortega 815 (HUAA). Calvillo: Ladera al W de la presa La Codorniz, Rosales-Carrillo 1435 (HUAA); $1 \mathrm{~km}$ al $\mathrm{N}$ de Jaltiche de Abajo, $21^{\circ} 47^{\prime} 12.5^{\prime \prime} \mathrm{N} 102^{\circ} 48^{\prime} 36.5^{\prime \prime} \mathrm{W}$, Sandoval-Ortega 963 (HUAA); $1 \mathrm{~km}$ por el libramiento Calvillo - Jalpa; Rosales-Carrillo 1451 (HUAA); Presa Los Sanates, al SE de presa Malpaso, Rosales-Carrillo 2130 (HUAA); Presa de los Serna, Rosales-Carrillo 1851 (HUAA); Presa Nueva, $1.5 \mathrm{~km}$ al N de Presa de los Serna, $21^{\circ} 49^{\prime} 16.6^{\prime \prime} \mathrm{N} 102^{\circ} 50^{\prime} 21.1^{\prime \prime} \mathrm{W}$, Sandoval-Ortega 850 (HUAA). Pabellón de Arteaga: Falda SE de Cerro Alto, a $4.6 \mathrm{~km}$ al NW deEl Garabato, 22 $05^{\prime} 27.0^{\prime \prime} \mathrm{N} 102^{\circ} 23^{\prime} 08.0^{\prime \prime} \mathrm{W}$, Sandoval-Ortega 883 (HUAA). San Francisco de los Romo: A 3.8 km al SW de Hacienda el Garabato, $22^{\circ} 04^{\prime} 15.2^{\prime \prime} \mathrm{N} 102^{\circ} 22^{\prime} 27.0^{\prime \prime} \mathrm{W}$, Sandoval-Ortega 837 (HUAA).

Amaranthus powellii S. Watson

Aguascalientes: Baldío del Fracc. Sta. Elena, 21 ${ }^{\circ} 52^{\prime} 06.0^{\prime \prime} \mathrm{N} 102^{\circ} 18^{\prime} 28.2^{\prime \prime} \mathrm{W}$, Esparza S. 115 (HUAA);

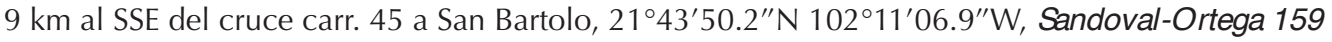
(HUAA); Presa El Muerto, al E de Peñuelas, Rosales-Carrillo 1338 (HUAA); Ciudad Universitaria,

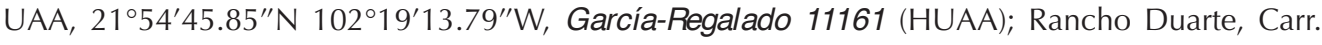
Ags. Calvillo, De la Cerda-Lemus 4791 (HUAA); a $2.5 \mathrm{~km}$ al NW de Calvillito, 21 ${ }^{\circ} 48^{\prime} 18.6^{\prime \prime} \mathrm{N}$ $102^{\circ} 10^{\prime} 14.5^{\prime \prime} \mathrm{W}$, Sandoval-Ortega 813a (HUAA). Asientos: $1.8 \mathrm{~km}$ al SE de Puerto de la Concepción, $22^{\circ} 11^{\prime} 21.7^{\prime \prime} \mathrm{N} 102^{\circ} 07^{\prime} 21.3^{\prime \prime} \mathrm{W}$, Sandoval-Ortega 800 (HUAA); Parque de Cactáceas, RosalesCarrillo 1250 (HUAA). Calvillo: Huerta El Chilarillo, arroyo Los Caballos, Nieto-Silva 64 (HUAA);

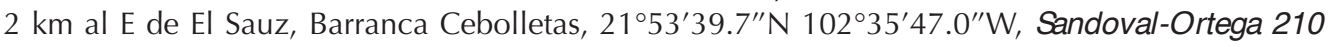


EXAMINED SPECIMENS: Continuation.

(HUAA); Arroyo Los Salates, Cañada Puente del Riego, 21 ${ }^{\circ} 50^{\prime} 50.1^{\prime \prime} \mathrm{N} 102^{\circ} 38^{\prime} 50.9^{\prime \prime} \mathrm{W}$, Sandoval-

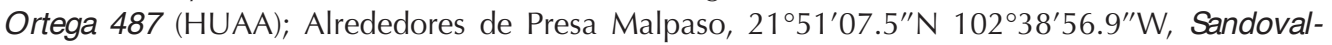
Ortega 575 (HUAA); $0.6 \mathrm{~km}$ al SE de Rancho Las Joyas, $21^{\circ} 46^{\prime} 40.1^{\prime \prime} \mathrm{N} 102^{\circ} 38^{\prime} 12.0^{\prime \prime} \mathrm{W}$, SandovalOrtega 889 (HUAA); Cerro la Iguana, 4.5km al NNW de El Sauz, 21 ${ }^{\circ} 54^{\prime} 56.6^{\prime \prime} \mathrm{N} 102^{\circ} 38^{\prime} 36.8^{\prime \prime} \mathrm{W}$, Sandoval-Ortega 897 (HUAA); Rancho Pensamiento, Rosales-Carrillo 1355 (HUAA); Río Malpaso, Rosales-Carrillo 1426 (HUAA); 25 km al W de Ags., carr. Ags-Calv., De la Cerda-Lemus 5657 (HUAA); Barranca la Botita, 215013.210239 02.6, Sandoval-Ortega 494a(HUAA); Presa Nueva,

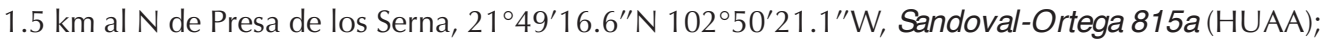
$2 \mathrm{~km}$ al NE de Malpaso, $21^{\circ} 52^{\prime} 23.6^{\prime \prime} \mathrm{N} 102^{\circ} 39^{\prime} 22.4^{\prime \prime} \mathrm{W}$, Sandoval-Ortega 569 (HUAA). El Llano: Arroyo el aguacero, a $2.5 \mathrm{~km}$ al NE de El Tule, $2^{\circ} 54^{\prime} 50.0^{\prime \prime} \mathrm{N} 101^{\circ} 54^{\prime} 25.7^{\prime \prime} \mathrm{W}$, Sandoval-Orte-

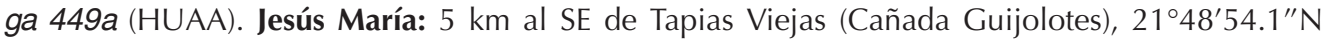
102'31'30.4"W, Sandoval-Ortega 459 (HUAA); Arroyo Mesa de Piedra, al E de los Muñoz, Rosales-Carrillo 1724 (HUAA). Pabellón de Arteaga: $\mathrm{N}$ de Ojo Zarco, Carretera a Asientos, De la Cerda-Lemus 5951 (HUAA). San José de Gracia: $3 \mathrm{~km}$ al NW de la cortina de la presa El Jo-

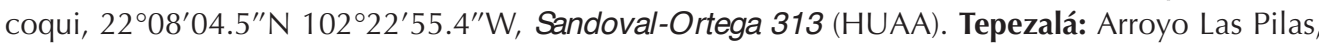
$22^{\circ} 15^{\prime} 32.7^{\prime \prime} \mathrm{N} 102^{\circ} 10^{\prime} 18.3^{\prime \prime} \mathrm{W}$, Sandoval-Ortega 341 (HUAA).

\section{Amaranthus spinosus $\mathrm{L}$.}

Calvillo: Las Pilas, $21^{\circ} 50^{\prime} 14.6^{\prime \prime} \mathrm{N} 102^{\circ} 46^{\prime} 14.6^{\prime \prime} \mathrm{W}$, Sandoval-Ortega 947 (HUAA).

\section{Amaranthus viridis $\mathrm{L}$.}

Aguascalientes: Avenida López mateos esquina Doctor Días de León, Ciudad de Aguascalientes, $21^{\circ} 52^{\prime} 44.8^{\prime \prime} \mathrm{N} 102^{\circ} 17^{\prime} 29.6^{\prime \prime} \mathrm{W}$, Sandoval-Ortega 1000 (HUAA).

Atriplex muricata Humb. \& Bonpl. ex Willd.

Aguascalientes: $7.2 \mathrm{~km}$ al NE del Instituto Tecnológico de EL Llano, $21^{\circ} 47^{\prime} 02.1^{\prime \prime} \mathrm{N} 102^{\circ} 09^{\prime} 38.2^{\prime \prime} \mathrm{W}$, Sandoval-Ortega M.H. 805 (HUAA). Asientos: Presa el Llavero, $22^{\circ} 04^{\prime} 00.2^{\prime \prime} \mathrm{N} 102^{\circ} 05^{\prime} 48.0^{\prime \prime} \mathrm{W}$, Sandoval-Ortega M.H. 110 (HUAA). Rincón de Romos: Arroyo la Boquilla, $500 \mathrm{~m}$ al W de La Boquilla, $22^{\circ} 15^{\prime} 50.6^{\prime \prime} \mathrm{N} 102^{\circ} 22^{\prime} 37.9^{\prime \prime} \mathrm{W}$ San José de Gracia: $3 \mathrm{~km}$ al $\mathrm{N}$ de San José de Gracia, carr. A la Congoja, $22^{\circ} 10^{\prime} 25.7^{\prime \prime} \mathrm{N} 102^{\circ} 25^{\prime} 10.5^{\prime \prime} \mathrm{W}$, Sandoval-Ortega M.H. 583 (HUAA); Extremo NE de San Antonio de los Ríos, $22^{\circ} 10^{\prime} 04.7^{\prime \prime} \mathrm{N} 102^{\circ} 28^{\prime} 03.0^{\prime \prime} \mathrm{W}$, Sandoval-Ortega M.H. 603 (HUAA).

Atriplex semibaccata R. Brown

Asientos: San Gil, extremo NW, García-Regalado 3905 (HUAA); $4 \mathrm{~km}$ al SE de Asientos,

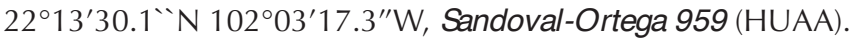

Atriplex suberecta I. Verd.

Aguascalientes: Universidad Autónoma de Aguascalientes, $21^{\circ} 54^{\prime} 55.3^{\prime \prime} \mathrm{N} 102^{\circ} 19^{\prime} 12.6^{\prime \prime} \mathrm{W}$, Sandoval-Ortega 956 (HUAA) Calvillo: Alrededores de Presa Malpaso, $21^{\circ} 51^{\prime} 07.5^{\prime \prime} \mathrm{N} 102^{\circ} 38^{\prime} 56.9^{\prime \prime} \mathrm{W}$, Sandoval-Ortega 572 (HUAA). Pabellón de Artega: $1 \mathrm{~km}$ al W de Santiago, $22^{\circ} 07^{\prime} 00.4^{\prime \prime} \mathrm{N}$ $102^{\circ} 20^{\prime} 52.9^{\prime \prime} \mathrm{W}$, Sandoval-Ortega 608 (HUAA). Rincón de Romos: Orilla de carretera paralela a

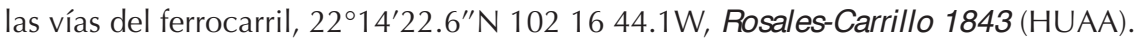

\section{Chenopodium album $\mathrm{L}$.}

Aguascalientes: a $2.5 \mathrm{~km}$ al NW de Calvillito, $21^{\circ} 48^{\prime} 18.6^{\prime \prime} \mathrm{N} 102^{\circ} 10^{\prime} 14.5^{\prime \prime} \mathrm{W}$, Sandoval-Ortega 810 (HUAA); lado W de Cd. Universitaria UAA, Rosales-Carrillo 1143 (HUAA); Ejido de la Guayana, De la Cerda-Lemus 4628 (HUAA); Ejido Sta. Cruz, $3 \mathrm{~km}$ al W de Jaltomate, De la Cerda-Lemus 4756 (HUAA); Ejido El Montoro, De la Cerda-Lemus 5058 (HUAA); Rancho El Padre, San Rafaél El Chico, De la Cerda-Lemus 5405 (HUAA). Asientos: 3 km al N de El Llavero, $22^{\circ} 04^{\prime} 08.8^{\prime \prime} \mathrm{N} 102^{\circ} 05^{\prime} 59.5^{\prime \prime} \mathrm{W}$, Sandoval-Ortega 105 (HUAA); San Gil, De la Cerda-Lemus 4774 (HUAA). Calvillo: Presa Los Adobes, Rosales-Carrillo 1601 (HUAA); Cañada Cebolletas, RosalesCarrillo 2218 (HUAA); Palo Alto, De la Cerda-Lemus 6289 (HUAA). Cosío: Tanque La Punta, al E 
EXAMINED SPECIMENS: Continuation.

del Poblado La punta, Rosales-Carrillo 2052 (HUAA). Jesús María: 3 km al SW de Tapias Viejas, $21^{\circ} 49^{\prime} 57.0^{\prime \prime} \mathrm{N} 102^{\circ} 33^{\prime} 42.9^{\prime \prime} \mathrm{W}$, Sandoval-Ortega 652 (HUAA); Arroyo La Gloria, al SW de presa

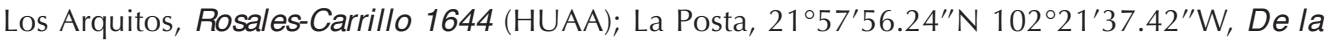
Cerda-Lemus 4724 (HUAA); El Rinconcito, $23 \mathrm{~km}$ al W de Aguascalientes, De la Cerda-Lemus

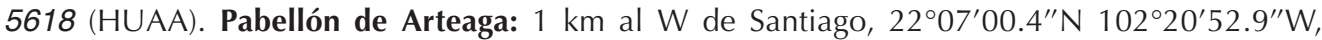
Sandoval-Ortega 612 (HUAA); Libramiento a Luis Moya, Frente a Colonia Campestre, RosalesCarrillo 1471 (HUAA); 2 km al N del Entronque a Pabellón, Siqueiros-Delgado 2589 (HUAA); $\mathrm{N}$ de Ojo Zarco, Carretera a Asientos, De la Cerda-Lemus 5938 (HUAA). Rincón de Romos: Barranca Sierpens, al W de Las Camas, Rosales-Carrillo 1238 (HUAA); Las Norias de Salitrillo, De la Cerda-Lemus 4696 (HUAA); Rancho El Banco, Lado E del Saucillo, De la Cerda-Lemus 4851 (HUAA); El Rocío, $3 \mathrm{~km}$ al E de Valle de Las Delicias, De la Cerda-Lemus 4951 (HUAA). San Francisco de Los Romo: Granja Las Moras, $1.5 \mathrm{~km}$ al $\mathrm{N}$ del entronque Pabellón- Emiliano Zapata, De la Cerda-Lemus 4821 (HUAA); Rancho La Alberca, $1 \mathrm{~km}$ al N del Libramiento, De la Cerda-Lemus 4831 (HUAA). San José de Gracia: Mesa Montoro, Rosales-Carrillo 1721 (HUAA); 1 km al E de La Congoja, Rosales-Carrillo 1894 (HUAA); El Zepo, Sierra San Blas de Pabellón, García-Regalado 2646 (HUAA).

\section{Chenopodium berlandieri Moq.}

Calvillo: $25 \mathrm{~km}$ al W de Ags., carr. Aguascalientes-Calvillo, De la Cerda-Lemus 5659 (HUAA); Palo Alto, De la Cerda-Lemus 6289 (HUAA); $0.5 \mathrm{~km}$ al W de la Barranca El Pilar, $22^{\circ} 04^{\prime} 14.7^{\prime \prime} \mathrm{N}$ $102^{\circ} 43^{\prime} 47.6^{\prime \prime} \mathrm{W}$, Sandoval-Ortega 134 (HUAA); Presa Los Adobes, Rosales-Carrillo 1601 (HUAA); Presa de los Serna, Rosales-Carrillo 1849 (HUAA); Cañada Cebolletas, Rosales-Carrillo 2220

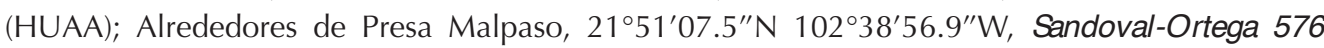

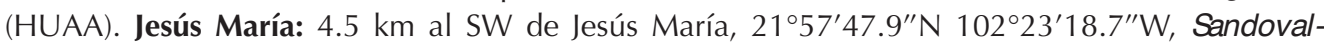
Ortega 281 (HUAA). Pabellón de Arteaga: $1 \mathrm{~km}$ al O de Santiago, 22 $07^{\circ} 00.4^{\prime \prime} \mathrm{N} 102^{\circ} 20^{\prime} 52.9^{\prime \prime} \mathrm{W}$, Sandoval-Ortega 612 (HUAA). San José de Gracia: $3 \mathrm{~km}$ al NW de la cortina de la presa El Jocoqui, $22^{\circ} 08^{\prime} 04.5^{\prime \prime} \mathrm{N} 102^{\circ} 22^{\prime} 55.4^{\prime \prime} \mathrm{W}$, Sandoval-Ortega 314 (HUAA).

Chenopodium fremontii S. Watson

Aguascalientes: $8 \mathrm{~km}$ al NE de Calvillito, De la Cerda-Lemus 5882 (HUAA; IEB). Pabellón de Arteaga: Faldas del Cerro El Chiquihuite, Rosales \& Croce 196 (HUAA). San Francisco de Los Romo: a $4.7 \mathrm{~km}$ al W de haciedna el Garabato, $22^{\circ} 04^{\prime} 57.8^{\prime \prime} \mathrm{N} 102^{\circ} 23^{\prime} 10.8^{\prime \prime} \mathrm{W}$, Sandoval-Ortega 821

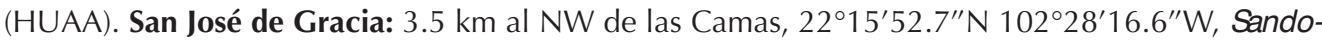
val-Ortega 192 (HUAA).

Chenopodiastrum murale (L.) S. Fuentes, Uotila \& Borsch

Aguascalientes: El Sabinal $2 \mathrm{~km}$ al S de El Salto de los Salado, $21^{\circ} 44^{\prime} 28.15^{\prime \prime} \mathrm{N} 102^{\circ} 22^{\prime} 4.47^{\prime \prime} \mathrm{W}$, Sandoval-Ortega 532 (HUAA); Ejido Sta. Cruz, $3 \mathrm{~km}$ al W de Jaltomate, De la Cerda-Lemus 4757 (HUAA); 1.5 km al E de Ags. (Ojo de Palmitas), De la Cerda-Lemus 5877 (HUAA). Asientos: Cerro al SE del Parque de Cactáceas, Rosales-Carrillo 1772 (HUAA). Pabellón de Arteaga: Centro Experimental INIFAP, De la Cerda-Lemus s.n. (HUAA). Rincón de Romos: El Panojal, Rosales-Carrillo 1830 (HUAA). San Francisco de Los Romo: a 4.7 km al W de Hacienda El Garabato, 22 $04^{\prime} 57.8^{\prime \prime} \mathrm{N}$ $102^{\circ} 23^{\prime} 10.8^{\prime \prime}$ W, Sandoval-Ortega 828 (HUAA). San José de Gracia: Barranca al extremo NE de San José de Gracia, 22 $2^{\circ} 9^{\prime} 20.6^{\prime \prime} \mathrm{N} 102^{\circ} 24^{\prime} 35.3^{\prime \prime} \mathrm{W}$, Sandoval-Ortega 592 (HUAA).

Dysphania ambrosioides (L.) Mosyakin \& Clemants

Aguascalienes: Casa particular, Croce M. s.n. (HUAA). Calvillo: Palo Alto, De la Cerda-Lemus 6289 (HUAA); Cañada Cebolletas, Rosales-Carrillo 2220 (HUAA); Arroyo Los Salates, Cañada Puente

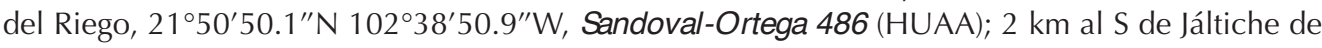
arriba, $21^{\circ} 45^{\prime} 19.3^{\prime \prime} \mathrm{N} 102^{\circ} 47^{\prime} 36.7^{\prime \prime} \mathrm{W}$, Sandoval-Ortega 856 (HUAA); $1.4 \mathrm{~km}$ al SW de la Labor, $21^{\circ} 57^{\prime} 23.4^{\prime \prime} \mathrm{N} 102^{\circ} 43^{\prime} 32.1^{\prime \prime} \mathrm{W}$, Sandoval-Ortega 635 (HUAA); $700 \mathrm{~m}$ al NW de El Sauz de la

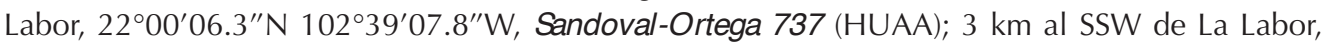


EXAMINED SPECIMENS: Continuation.

$21^{\circ} 56^{\prime} 20.3^{\prime \prime} \mathrm{N} 102^{\circ} 42^{\prime} 48.1^{\prime \prime} \mathrm{W}$, Sandoval-Ortega 643 (HUAA); Alrededores de Presa Malpaso, $21^{\circ}$ 51'07.5”N 10238 56.9W, Sandoval-Ortega 574 (HUAA). Cosío: Tanque La Punta, al E del Poblado La punta, $21^{\circ} 19^{\prime} 16^{\prime \prime} \mathrm{N} 102^{\circ} 17^{\prime} 09^{\prime \prime} \mathrm{W}$, Rosales-Carrillo 2039 (HUAA); Jesús María: 3 km al SW de Tapias Viejas, $21^{\circ} 49^{\prime} 57.0^{\prime \prime} \mathrm{N} 102^{\circ} 33^{\prime} 42.9^{\prime \prime} \mathrm{W}$, Sandoval-Ortega 653 (HUAA). San José de Gracia: Mesa Montoro, Rosales Carrillo 2111 (HUAA); 3 km al NW de la cortina de la presa El Jocoqui, $22^{\circ} 08^{\prime} 04.5^{\prime \prime} \mathrm{N} 102^{\circ} 22^{\prime} 55.4^{\prime \prime} \mathrm{W}$, Sandoval-Ortega 310 (HUAA); $700 \mathrm{~m}$ al E de la cortina de la Presa Calles, $22^{\circ} 08^{\prime} 17.0^{\prime \prime} \mathrm{N} 102^{\circ} 24^{\prime} 47.0^{\prime \prime} \mathrm{W}$, Sandoval-Ortega 510 (HUAA); Barranca el Carrizal a $4.5 \mathrm{~km}$ al NW de San Antonio de Los Ríos, $22^{\circ} 11^{\prime} 05.8^{\prime \prime} \mathrm{N} 102^{\circ} 30^{\prime} 23.5^{\prime \prime} \mathrm{W}$, Sandoval-Ortega 555 (HUAA); Margen NW de la Presa Calles, $22^{\circ} 09^{\prime} 42.1^{\prime \prime} \mathrm{N} 102^{\circ} 27^{\prime} 22.1^{\prime \prime} \mathrm{W}$, Sandoval-Ortega 564 (HUAA); Cañada $1.2 \mathrm{~km}$ al SE de Potrero de Los Lopez, 22 ${ }^{\circ} 04^{\prime} 51.0^{\prime \prime} \mathrm{N} 102^{\circ} 30^{\prime} 53.8^{\prime \prime} \mathrm{W}$, Sandoval-Ortega 559 (HUAA); Extremo NE de San Antonio de los Ríos, $22^{\circ} 10^{\prime} 04.7^{\prime \prime} \mathrm{N} 102^{\circ} 28^{\prime} 03.0^{\prime \prime} \mathrm{W}$, Sandoval-Ortega

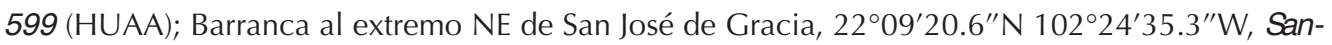
doval-Ortega 594 (HUAA).

Dysphania carinata (R. Brown) Mosyakin \& Clemants

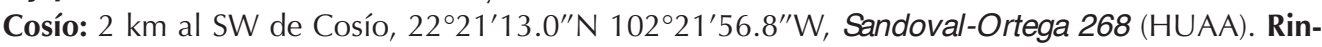

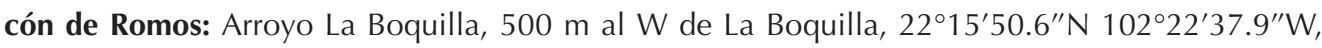
Martínez-Ramírez 2498 (HUAA). Tepezalá: Presa Mesillas, a $1 \mathrm{~km}$ al SE de Mesillas, $22^{\circ} 18^{\prime} 48.1^{\prime \prime} \mathrm{N}$ $102^{\circ} 09^{\prime} 40.7^{\prime \prime} \mathrm{W}$, Martínez-Ramirez 2404 (HUAA); Arroyo al SE de Mesillas, $22^{\circ} 18^{\prime} 40.29^{\prime \prime} \mathrm{N}$ $102^{\circ} 09^{\prime} 47.75^{\prime \prime} \mathrm{W}$, Rosales-Carrillo 3062 (HUAA).

Dysphania graveolens (Willd.) Mosyakin \& Clemants

Aguascalientes: Rancho el Refugio, $21^{\circ} 47^{\prime} 37.3^{\prime \prime} \mathrm{N} 102^{\circ} 15^{\prime} 10.2^{\prime \prime} \mathrm{W}$, Sandoval-Ortega 869 (HUAA); $8 \mathrm{~km}$ al E de Aguascalientes (1 km al NE de Calvillito), De la Cerda-Lemus 5881 (HUAA); Cerro Los Gallos, Rosales-Carrillo 2005 (HUAA); Bordos permanentes al SW de Tanque de Los Jimenez, Rosales-Carrillo 1948 (HUAA). Asientos: Presa el Llavero, $22^{\circ} 04^{\prime} 00.2^{\prime \prime} \mathrm{N} 102^{\circ} 05^{\prime} 48.0^{\prime \prime} \mathrm{W}$, Sandoval-Ortega 109 (HUAA); Bordo al extremo SW de Plutarco Elías Calles; 22 $12^{\prime} 32.7^{\prime \prime} \mathrm{N}$ $102^{\circ} 03^{\prime} 25.7^{\prime \prime}$ W, Sandoval-Ortega 249 (HUAA); Bimbaletes, Villalobos s.n. (HUAA). Calvillo: 4 km al NW del Temazcal, De la Cerda-Lemus 6229 (HUAA); Barranca el Sauz, García-Regalado 4705 (HUAA); La Playita, carr. Aguascalientes-Calvillo, García-Regalado 5042 (HUAA); Los Alisos, Siqueiros-Delgado 1918 (HUAA; IEB); $1 \mathrm{~km}$ al SE de la entrada al Rancho Las Cuatas,

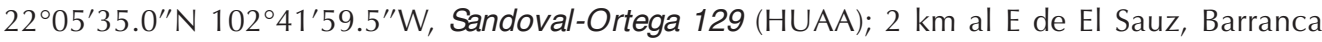
Cebolletas, 21 ${ }^{\circ} 53^{\prime} 39.7^{\prime \prime} \mathrm{N} 102^{\circ} 35^{\prime} 47.0^{\prime \prime} \mathrm{W}$, Sandoval-Ortega 206 (HUAA); Mesa El Ocote, km al NW de El Terrero del refugio, García-Regalado 4848 (HUAA; MEXU). Cosío: 2 km al SW de Cosío, $22^{\circ} 21^{\prime} 13.0^{\prime \prime} \mathrm{N} 102^{\circ} 18^{\prime} 56.8^{\prime \prime} \mathrm{W}$, Sandoval-Ortega 264 (HUAA). El Llano: Rancho San Miguel de Salvador, Esparza S. 24 (HUAA). Jesús María: $1.3 \mathrm{~km}$ al NNW de la Tomatina, 21 ${ }^{\circ} 54^{\prime} 49.2^{\prime \prime} \mathrm{N}$ $102^{\circ} 25^{\prime} 05.7^{\prime \prime} \mathrm{W}$, Sandoval-Ortega 237 (HUAA); $1.3 \mathrm{~km}$ al SW de la Tomatina, 21 ${ }^{\circ} 53^{\prime} 50.6^{\prime \prime} \mathrm{N}$ $102^{\circ} 25^{\prime} 37.0^{\prime \prime} \mathrm{W}$, Sandoval-Ortega 242 (HUAA); Cortina de la Presa El jarabe a $4.5 \mathrm{~km}$ al SE

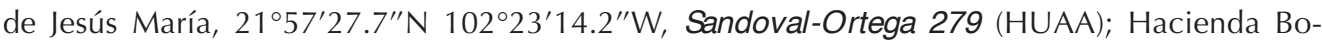
badillo, Cerro el Colorín, Siqueiros-Delgado 2485 (HUAA). Pabellón de Arteaga: Terracería El Garabato-Mesa el Pino a $4.3 \mathrm{~km}$ al W de Garabatos, $22^{\circ} 04^{\prime} 56.9^{\prime \prime} \mathrm{N} 102^{\circ} 22^{\prime} 58.8^{\prime \prime} \mathrm{W}$, Sandoval-Ortega 290 (HUAA). San Francisco de Los Romo: a 4.7 km al W de hacienda el Garabato,

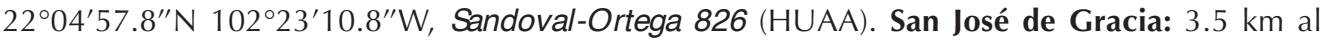
NW de Las Camas, 22 ${ }^{\circ} 15^{\prime} 52.7^{\prime \prime} \mathrm{N} 102^{\circ} 28^{\prime} 16.6^{\prime \prime} \mathrm{W}$, Sandoval-Ortega 189 (HUAA); $700 \mathrm{~m}$ al W

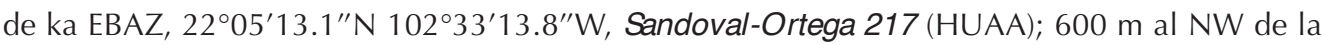
EBAZ, $22^{\circ} 05^{\prime} 35.9^{\prime \prime} \mathrm{N} 102^{\circ} 33^{\prime} 34.9^{\prime \prime} \mathrm{W}$, Sandoval-Ortega 233 (HUAA); $3 \mathrm{~km}$ al NW de la cortina de la presa El Jocoqui, $22^{\circ} 08^{\prime} 04.5^{\prime \prime} \mathrm{N} 102^{\circ} 22^{\prime} 55.4^{\prime \prime} \mathrm{W}$, Sandoval-Ortega 318 (HUAA); Parte alta de Barranca Juan Francisco, 22 $2^{\circ} 2^{\prime} 37.4^{\prime \prime} \mathrm{N} 102^{\circ} 37^{\prime} 56.7^{\prime \prime}$ W, Sandoval-Ortega 473 (HUAA); Mesa Montoro, Rosales-Carrillo 2107 (HUAA); Km 53 SSE carr. La Congoja-La Labor, Rosales-Carrillo 2153 (HUAA); alrededor de $1 \mathrm{~km}$ al E de la Congoja, Rosales-Carrillo 1893 (HUAA); El Agua Escondida, García-Regalado 2047 (HUAA). Tepezalá: 1 km al W de Puerto de la Concepción, 22 $11^{\prime} 28^{\prime \prime} \mathrm{N} 102^{\circ} 08^{\prime} 53^{\prime \prime} \mathrm{W}$, De la Cerda-Lemus 2214 (HUAA). 
EXAMINED SPECIMENS: Continuation.

Froelichia interrupta (L.) Moq.

Aguascalientes: Rancho el Refugio, $21^{\circ} 47^{\prime} 37.3^{\prime \prime} \mathrm{N} 102^{\circ} 15^{\prime} 10.2^{\prime \prime} \mathrm{W}$, Sandoval-Ortega 873 (HUAA); Ciudad Universitaria UAA, Rosales-Carrillo 1147 (HUAA); $2 \mathrm{~km}$ al S de la Hacienda San Bartolo, Rosales-Carrillo 1321 (HUAA); Al E de San Pedro Cieneguilla, Lado E del Río San Pedro, Rosales-Carillo 1930 (HUAA). Calvillo: $700 \mathrm{~m}$ al NW de El Sauz de la Labor, 22 $00^{\prime} 06.3^{\prime \prime} \mathrm{N}$ $102^{\circ} 39^{\prime} 07.8^{\prime \prime} \mathrm{W}$, Sandoval-Ortega 738 (HUAA); $1 \mathrm{~km}$ al S de La Fragua, Rosales-Carrillo 3821 (HUAA); Cerro Blanco, Rosales-Carrillo 1870 (HUAA). Cosío: $1.5 \mathrm{~km}$ al W del Salero, Rosales \&

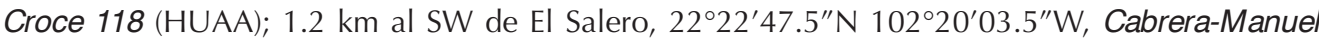
598 (HUAA). El Llano: Rancho Los Robles, $1.5 \mathrm{~km}$ al NE de Ojo de Agua de Crucitas, 21 ${ }^{\circ} 58^{\prime} 04.1^{\prime \prime} \mathrm{N}$ 101 ${ }^{\circ} 6^{\prime} 12.1^{\prime \prime} \mathrm{W}$, Sandoval-Ortega 666 (HUAA). Pabellón de Arteaga: $1 \mathrm{~km}$ al W de Santiago,

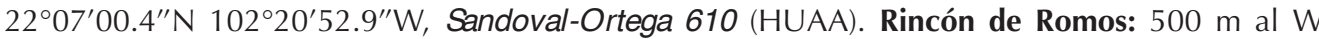
de La Punta, $22^{\circ} 19^{\prime} 16^{\prime \prime} \mathrm{N} 102^{\circ} 17^{\prime} 55^{\prime \prime}$ W, Serra-Muñoz 858 (HUAA); $3 \mathrm{~km}$ al SW de La punta, $22^{\circ} 18^{\prime} 39^{\prime \prime} \mathrm{N} 102^{\circ} 19^{\prime} 11^{\prime \prime} \mathrm{W}$, Sierra-Muñoz 848 (HUAA); Barranca Sierpens, al W de Las Camas, Rosales-Carrillo 1216 (HUAA); Lado W del poblado La Escalera, Rosales-Carrillo 1174 (HUAA); Cañada al S de Mesa Verde, Rosales \& Croce 84 (HUAA). San José de Gracia: 3 km al N de San José de Gracia, carr. a la Congoja, $22^{\circ} 10^{\prime} 25.7^{\prime \prime} \mathrm{N} 102^{\circ} 25^{\prime} 10.5^{\prime \prime} \mathrm{W}$, Sandoval-Ortega 581 (HUAA); Barranca al extremo NE de San José de Gracia, $22^{\circ} 09^{\prime} 20.6^{\prime \prime} \mathrm{N} 102^{\circ} 24^{\prime} 35.3^{\prime \prime} \mathrm{W}$, Sandoval-Ortega 596 (HUAA); Presa Jocoqui, Gonzales-Adame 385 (HUAA); 2 km al SE de Arroyo Viejo Camino a Potrero de Los López, Rosales-Carrillo 1281 (HUAA); $1 \mathrm{~km}$ al W de la Presa Jocoqui, De la CerdaLemus 2470 (HUAA); Arroyo viejo, al SW de paredes, Rosales-Carrillo 1263 (HUAA).

\section{Gomphrena serrata L.}

Aguascalientes: Bordos permanentes al SW de Tanque de Los Jimenez, Rosales-Carrillo 1951 (HUAA); Al W de Cieneguilla, Lado E del Río San Pedro, Rosales-Carrillo 1927 (HUAA); Rumbo al Cerro de Los Gallos, Rosales-Carrillo 1982 (HUAA); Cerro El Picacho, De la Cerda-Lemus \& Gar-

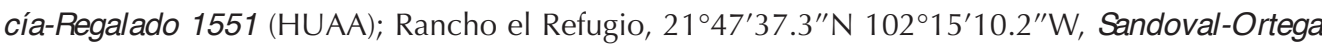
866 (HUAA); Ciudad de Aguascalientes, Weeke Z. 41 (HUAA); 200 m del entronque carr. Aguascalientes-Encarnación de Díaz, Cerro Los Gallos, De la Cerda-Lemus 5803 (HUAA); Extremo W del Ocote, De la Cerda-Lemus 5776 (HUAA); 1.5 km al E de Ags. (Ojo de Palmitas), De la Cerda-Lemus 5876 (HUAA); $1 \mathrm{~km}$ al S de Rancho El Catorce, carr. Aguascalientes-Villa Hidalgo, De la CerdaLemus 5790 (HUAA); Ladera N, Cerro El Picacho, De la Cerda-Lemus \& García-Regalado 1029 (HUAA); Ladera E, Cerro El Picacho, García-Regalado 2666 (HUAA); $8.1 \mathrm{~km}$ al E de Aguascalientes (1 km al NE de Calvillito), De la Cerda-Lemus 5896 (HUAA); Cañada al W de la presa El Muerto, Rosales-Carrillo 1838 (HUAA); Presa El Muerto, al E de Peñuelas, Rosales-Carrillo 1342 (HUAA); Entre presa y la Hacienda San Bartolo, 21 ${ }^{\circ} 43^{\prime} 87^{\prime \prime} \mathrm{N} 102^{\circ} 13^{\prime} 64^{\prime \prime}$ W, Rosales-Carrillo 1315 (HUAA); 1 km al SW de José María Morelos (Cañada Honda), Rosales-Carrillo 1245 (HUAA); El Límite, Rosales \& Croce 170 (HUAA); Parte W de ciudad universitaria, UAA, Rosales-Carrillo 1146 (HUAA);

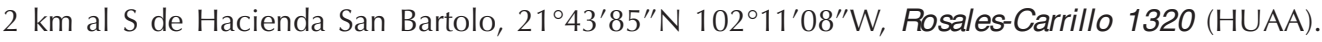
Asientos: Parque de Cactáceas, Rosales-Carrillo 1249 (HUAA). Calvillo: Barranca el Sauz, GarcíaRegalado 4704 (HUAA); Cerro Blanco, Rosales-Carrillo 1877 (HUAA); La Pitayita (Entre el Salitre y las Tinajas), De la Cerda-Lemus 5108 (HUAA); 4 km al NW del Temazcal, Rosales-Carrillo 1330 (HUAA); 1 km al NW de La Labor, García-Regalado 448 (HUAA); Entronque Rio Gil-Carr. a Calvillo, García-Regalado 4266 (HUAA); Límite Jesús María-Calvillo, García-Regalado 4289 (HUAA); Presa Los Serna, Rosales-Carrillo 1850 (HUAA); 2 km al N de Presa de Los Serna, García-Regalado 4873 (HUAA); 2.7 km al S de la presa Los Serna, Barranca 3 Cruces, García-Regalado 4746

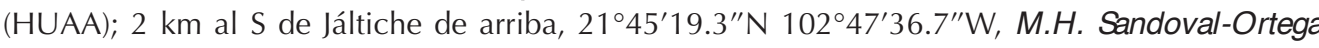
853 (HUAA); Mesa San Tadeo, Squeiros-Delgado 1801 (HUAA); Carr. Aguascalientes-Calvillo km 26.5, Nieto-SIlva 3 (HUAA), Camarillo C. 6 (HUAA); 4 km al SE de Malpaso, De la Cerda-Lemus \& García-Regalado 155 (HUAA); 2 km al S de Malpaso, De la Cerda-Lemus \& García-Regalado 881 (HUAA); Alrededor de 1 km por el libramiento Calvillo-Jalpa, Rosales-Carrillo 1446 (HUAA); Río Gil, Rosales-Carrillo 1360 (HUAA); Rancho Pensamiento, Rosales-Carrillo 1351 (HUAA); Cañada Arroyo Hondo, De la Cerda-Lemus \& García-Regalado 1570 (HUAA); 25 km al W de Aguasca- 
EXAMINED SPECIMENS: Continuation.

lientes, carr. Aguascalientes-Calvillo, De la Cerda-Lemus 5665 (HUAA); Ladera W de presa La Codorniz, Rosales-Carrillo 1437 (HUAA); Los Alisos, Rosales-Carrillo 1301 (HUAA); Límite Aguascalientes-Zacatecas, al SW del Estado, Rosales-Carrillo 1454 (HUAA). Cosio: $1.2 \mathrm{~km}$ al SW de El

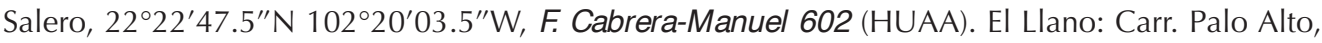
Frente a Sabro Pollo, Rosales \& Croce 27 (HUAA); $3 \mathrm{~km}$ al N de Ojo de Agua de Crucitas, Rosales \& Croce 20 (HUAA). Jesús María: $1.3 \mathrm{~km}$ al SW de la Tomatina, 21 ${ }^{\circ} 53^{\prime} 50.6^{\prime \prime} \mathrm{N} 102^{\circ} 25^{\prime} 37.0^{\prime \prime} \mathrm{W}$, Sandoval-Ortega 243 (HUAA); 8 km al S de Tapias Viejas, García-Regalado 4472 (HUAA); 3 km al SW de Tapias Viejas, $21^{\circ} 49^{\prime} 57.0^{\prime \prime} \mathrm{N} 102^{\circ} 33^{\prime} 42.9^{\prime \prime} \mathrm{W}$, Sandoval-Ortega 655 (HUAA); Curva doble A, Esparza S. 12 (HUAA); Alrededores de Milpillas de Arriba, Croce 20 (HUAA); Cerro La Presa, De la Cerda-Lemus 6239 (HUAA). Pabellón de Arteaga: Faldas del Cerro El Chiquihuite, Rosales \& Croce 214 (HUAA); Libramiento a Luis Moya, Frente a Colonia Campestre, Rosales-Carrillo 1470 (HUAA); N de Ojo Zarco, Carretera a Asientos, De la Cerda-Lemus 5960 (HUAA). Rincón de Romos: Barranca Sierpens, Rosales-Carrillo 1234 (HUAA); Lado W del poblado La Escalera, Rosales-Carrillo 1173 (HUAA); 4 km al SW de Pabellón de Hidalgo, De la Cerda-Lemus \& GarcíaRegalado 1618 (HUAA). San José de Gracia: Extremo N de la Cabecera Municipal del poblado de San José de Gracia, De la Cerda-Lemus 5711 (HUAA); Estación Biológica Agua Zarca, Rosales-Carrillo 1477 (HUAA); 2 km al SE de Arroyo Viejo Camino a Potrero de Los López, Rosales-Carrillo 1277 (HUAA); Al Sur de Potrero de Los López, Rosales-Carrillo 1285 (HUAA); Arroyo Viejo, al Sur de Paredes, Rosales-Carrillo 1256 (HUAA).

Guilleminea densa (Humb. \& Bonpl. ex Schult.) Moq.

Aguascalientes: a $7.2 \mathrm{~km}$ al NE del Instituto Tecnológico del Llano, $21^{\circ} 47^{\prime} 02.1^{\prime \prime} \mathrm{N} 102^{\circ} 09^{\prime} 38.2^{\prime \prime} \mathrm{W}$, Sandoval-Ortega 804 (HUAA); $1.5 \mathrm{~km}$ al E de Ags. (Ojo de Palmitas), De la Cerda-Lemus 5869 (HUAA); Entre presa y la Hacienda San Bartolo, 21 $44^{\prime} 13^{\prime \prime} \mathrm{N} 102^{\circ} 10^{\prime} 05^{\prime \prime}$ W, Rosales-Carrillo 1312 (HUAA); $1 \mathrm{~km}$ al S de Rancho El Catorce, carr. Aguascalientes-Villa Hidalgo, De la Cerda-Lemus 5799 (HUAA); AI W de Cieneguilla, Lado E del Río San Pedro, Rosales-Carrillo 1928 (HUAA); 1 km al S del Ejido Peñuelas, Rosales \& Croce 135 (HUAA); Ladera E, Cerro El Picacho, García-Regalado 2659 (HUAA); lado W de Cd. Universitaria UAA, Rosales-Carrillo 1148 (HUAA); El Límite, al S de Peñuelas, Rosales \& Croce 162 (HUAA); 200 m del entronque carr. Aguascalientes-Encarnación de Díaz, Cerro Los Gallos, De la Cerda-Lemus 5806 (HUAA); 8 km al E de Aguascalientes $(1 \mathrm{~km}$ al NE de Calvillito), De la Cerda-Lemus 5890 (HUAA); Salto de Ojocaliente, Croce s.n. (HUAA); $1 \mathrm{~km}$ al S de José María Morelos (Cañada Honda), Rosales-Carrillo 1241 (HUAA); Rumbo al Cerro de Los Gallos, Rosales-Carrillo 1969 (HUAA). Asientos: Cañada El Saucillo, Rosales-Carrillo 1786

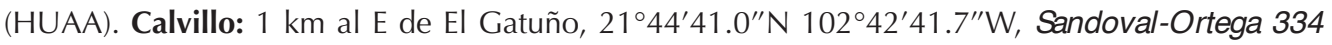
(HUAA); Antena de IUSACEL, al NW del Maguey, Rosales-Carrillo 1237 (HUAA); Presa al NW de Cerro Blanco, Rosales-Carrillo 1964 (HUAA); 4 km al NW del Temazcal, Rosales-Carrillo 1337 (HUAA); La Cieneguilla, límites con Jalisco, Rosales-Carrillo 1302 (HUAA). Cosío: $2 \mathrm{~km}$ al SO

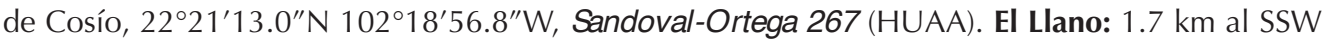
de Fco. Sarabia, $21^{\circ} 57^{\prime} 07.1^{\prime \prime} \mathrm{N} 102^{\circ} 01^{\prime} 20.0^{\prime \prime} \mathrm{W}$, Sandoval-Ortega 751 (HUAA); $1.5 \mathrm{~km}$ al SW de Fco. Sarabia, $21^{\circ} 57^{\prime} 21.9^{\prime \prime} \mathrm{N} 102^{\circ} 01^{\prime} 43.0^{\prime \prime} \mathrm{W}$, Sandoval-Ortega 756 (HUAA); $3 \mathrm{~km}$ al N de Ojo de Agua de Crucitas, Rosales \& Croce 24a (HUAA). Jesús María: El Rinconcito, 23 km al W de Aguascalientes, De la Cerda-Lemus 5636 (HUAA); Presa Abelardo Rodriguez, Siqueiros-Delgado 2562 (HUAA); San Isidro, carr. Calvillo-Tapias Viejas, De la Cerda-Lemus 5756 (HUAA); Cerro La Presa, $22^{\circ} 04^{\prime} 76^{\prime \prime} \mathrm{N} 102^{\circ} 20^{\prime} 84^{\prime \prime} \mathrm{W}$, De la Cerda-Lemus 6249 (HUAA). Pabellón de Arteaga: Libramiento a Luis Moya, Frente a Colonia Campestre, Rosales-Carrillo 1466 (HUAA); Faldas del Cerro El Chiquihuite, Rosales \& Croce 216 (HUAA). Rincón de Romos: Cañada al S de la Mesa Verde, Rosales \& Croce 82 (HUAA); Barranca Sierpens, al W de Las Camas, Rosales-Carrillo 1227 (HUAA); Lado W del poblado La Escalera, Rosales-Carrillo 1264 (HUAA); Barranca Sierpens, al W de Las Camas, Rosales-Carrillo 1186 (HUAA). San Francisco de Los Romo: a 4.7 km al W de Hacienda el Garabato, $23^{\circ} 04^{\prime} 57.8^{\prime \prime} \mathrm{N} 102^{\circ} 23^{\prime} 0.8^{\prime \prime} \mathrm{W}$, Sandoval-Ortega 831 (HUAA). San José de Gracia: 3 km al NW de la cortina de la presa El Jocoqui, $22^{\circ} 08^{\prime} 04.5^{\prime \prime} \mathrm{N} 102^{\circ} 22^{\prime} 55.4^{\prime \prime} \mathrm{W}$, Sandoval-Ortega 308 (HUAA); 3 km al N de San José de Gracia, carr. a la Congoja, $22^{\circ} 10^{\prime} 25.7^{\prime \prime} \mathrm{N} 102^{\circ} 25^{\prime} 10.5^{\prime \prime} \mathrm{W}$, Sandoval-Ortega 
EXAMINED SPECIMENS: Continuation.

582 (HUAA); 2 km al SW de la Congoja, Rosales-Carrillo 1377 (HUAA); Estación Biológica Agua Zarca, Rosales \& Castañeda 1549 (HUAA); 1 km al NW de la presa Jocoqui, De la Cerda \& García 1063 (HUAA); Ladera E. después de San Antonio de los Ríos, Rumbo a la Congoja, Rosales-Carrillo 1156 (HUAA); 2 km al SE de Arroyo Viejo Camino a Potrero de Los López, Rosales-Carrillo 1274 (HUAA); Mesa Montoro, Rosales-Carrillo 2126 (HUAA); Al Sur de Potrero de Los López, RosalesCarrillo 1284 (HUAA); Arroyo Viejo, al S de paredes, Rosales-Carrillo 1253 (HUAA).

Iresine diffusa Humb. \& Bonpl. ex Willd.

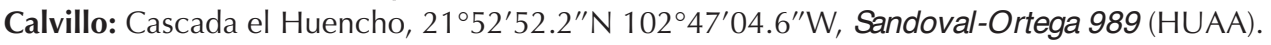

Iresine grandisStandl.

Calvillo: $1.7 \mathrm{~km}$ al W de El Gatuño, $21^{\circ} 44^{\prime} 25.6^{\prime \prime} \mathrm{N}, 102^{\circ} 44^{\prime} 1.6^{\prime \prime} \mathrm{W}$, Araiza-Arvilla 589 (HUAA), García-Regalado 6486 (HUAA); Ladera vertiente N, Los Alisos, Rosales-Carrillo 3874 (HUAA).

Iresine hartmanii Uline

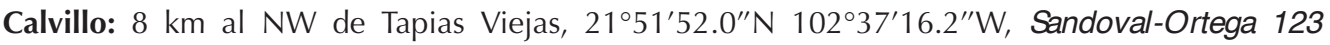

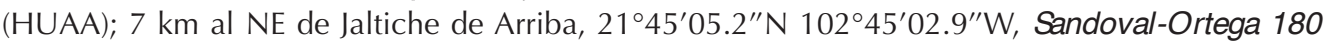

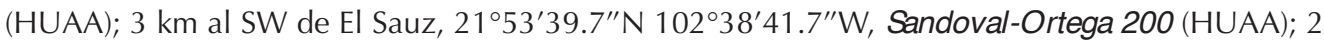
$\mathrm{km}$ al NE de Malpaso, 21 $52^{\prime} 23.6^{\prime \prime} \mathrm{N} 102^{\circ} 39^{\prime} 22.4^{\prime \prime} \mathrm{W}$, Sandoval-Ortega 568 (HUAA); Alrededores de Presa Malpaso, $21^{\circ} 51^{\prime} 07.5^{\prime \prime} \mathrm{N} 102^{\circ} 38^{\prime} 56.9^{\prime \prime} \mathrm{W}$, Sandoval-Ortega 580a (HUAA); Margenes de Presa La Ordeña Vieja, $21^{\circ} 56^{\prime} 53.3^{\prime \prime} \mathrm{N} 102^{\circ} 43^{\prime} 14.8^{\prime \prime} \mathrm{W}$, Sandoval-Ortega 639a (HUAA); Las Pilas, $21^{\circ} 50^{\prime} 14.6^{\prime \prime} \mathrm{N} 102^{\circ} 46^{\prime} 14.6^{\prime \prime} \mathrm{W}$, Sandoval-Ortega 950 (HUAA).

Iresine heterophylla Standl.

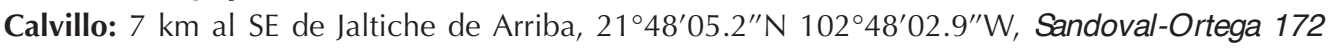

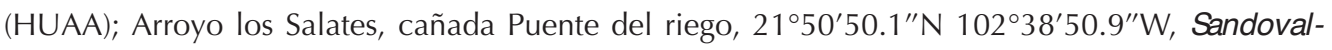
Ortega 493 (HUAA). Jesús María: Puentes Cuates, García-Regalado 3104 (HUAA); Cañada Puentes cuates, $21^{\circ} 51^{\prime} 26.5^{\prime \prime} \mathrm{N} 102^{\circ} 34^{\prime} 23.7^{\prime \prime} \mathrm{W}$, Sandoval-Ortega 909 (HUAA). San José de Gracia: Arroyo viejo, al SW de paredes, Rosales-Carrillo 1251 (HUAA).

Iresine latifolia (M. Martens \& Galeotti) Benth. \& Hook.

Calvillo: Arroyo Los Salates, Cañada Puente del Riego, 21 ${ }^{\circ} 50^{\prime} 50.1^{\prime \prime} \mathrm{N} 102^{\circ} 38^{\prime} 50.9^{\prime \prime} \mathrm{W}$, SandovalOrtega 490 (HUAA); 250 m al W de Balneario La Cueva, Martínez-Ramirez 2361 (HUAA); Verte-

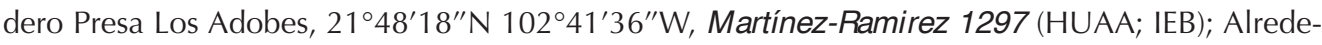
dores de Presa Malpaso, $21^{\circ} 51^{\prime} 07.5^{\prime \prime} \mathrm{N} 102^{\circ} 38^{\prime} 56.9^{\prime \prime} \mathrm{W}$, Sandoval-Ortega 580 (HUAA); Margenes de Presa La Ordeña Vieja, 21 $56^{\prime} 53.3^{\prime \prime} \mathrm{N} 102^{\circ} 43^{\prime} 14.8^{\prime \prime} \mathrm{W}$, Sandoval-Ortega 639 (HUAA); 2 km al NE de Malpaso, 21 ${ }^{\circ} 52^{\prime} 23.6^{\prime \prime} \mathrm{N} 102^{\circ} 39^{\prime} 22.4^{\prime \prime} \mathrm{W}$, Sandoval-Ortega 568a (HUAA). Cosío: Cerro Mesa verde al W del poblado la Punta, Rosales-Carrillo 2018 (HUAA). Jesús María: Barranca Montoro, a $4 \mathrm{~km}$ al N de Milpillas, 21 ${ }^{\circ} 59^{\prime} 44.0^{\prime \prime} \mathrm{N} 102^{\circ} 35^{\prime} 31.5^{\prime \prime} \mathrm{W}$, Sandoval-Ortega 539 (HUAA). San José de Gracia: $700 \mathrm{~m}$ al E de la cortina de la Presa Calles, $22^{\circ} 08^{\prime} 17.0^{\prime \prime} \mathrm{N} 102^{\circ} 24^{\prime} 47.0^{\prime \prime} \mathrm{W}$, Sandoval-Ortega 519 (HUAA); Tunel de Potrerillo, $1.5 \mathrm{~km}$ al E de Boca de Potrerillo, $22^{\circ} 14^{\prime} 04.90^{\prime \prime} \mathrm{N}$ $102^{\circ} 23^{\prime} 31.10^{\prime \prime} \mathrm{W}$, Sandoval-Ortega 589 (HUAA); Barranca al extremo NE de San José de Gracia, $22^{\circ} 09^{\prime} 20.6^{\prime \prime} \mathrm{N} 102^{\circ} 24^{\prime} 35.3^{\prime \prime} \mathrm{W}$, Sandoval-Ortega 590 (HUAA).

Iresine pringlei $\mathrm{S}$. Watson

Calvillo: $500 \mathrm{~m}$ al S del Rancho Cerro Blanco, $21^{\circ} 49^{\prime} 57.2^{\prime \prime} \mathrm{N} 102^{\circ} 48^{\prime} 43.2^{\prime \prime} \mathrm{W}$, Martínez-Robledo 180 (HUAA); Suroeste de Presa de Los Serna, Serra-Muñoz 86 (HUAA); El Tepozán, 11 km al W de San Tadeo, García-Regalado 3435 (HUAA). San José de Gracia: Cortina de la Presa Calles, $22^{\circ} 08^{\prime} 19.3^{\prime \prime} \mathrm{N} 102^{\circ} 24^{\prime} 52.5^{\prime \prime} \mathrm{W}$, Sandoval-Ortega 983 (HUAA).

Iresine schaffneri S. Watson

Aguascalientes: Cañada al W de Presa El Muerto, $21^{\circ} 52^{\prime} 19.44^{\prime \prime} \mathrm{N} 102^{\circ} 24^{\prime} 13.99^{\prime \prime} \mathrm{W}$, Rosales-Carrillo 
EXAMINED SPECIMENS: Continuation.

1841 (HUAA). Asientos: $1.8 \mathrm{~km}$ al SE de Puerto de la Concepción, $22^{\circ} 11^{\prime} 21.7^{\prime \prime} \mathrm{N} 102^{\circ} 07^{\prime} 21.3^{\prime \prime} \mathrm{W}$, Sandoval-Ortega 801a (HUAA). Calvillo: 3 km al SW de El Sauz, 21 ${ }^{\circ} 53^{\prime} 39.7^{\prime \prime} \mathrm{N} 102^{\circ} 38^{\prime} 41.7^{\prime \prime} \mathrm{W}$, Sandoval-Ortega 203 (HUAA); Arroyo Los Salates, Cañada Puente del Riego, 21 50'50.1"N $102^{\circ} 38^{\prime} 50.9^{\prime \prime} \mathrm{W}$, Sandoval-Ortega 488 (HUAA); Barranca Las Moras, $1 \mathrm{~km}$ al SE de la Cortina de

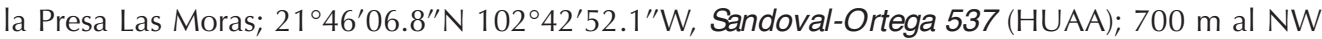
de El Sauz de la Labor, $22^{\circ} 00^{\prime} 06.3^{\prime \prime} \mathrm{N} 102^{\circ} 39^{\prime} 07.8^{\prime \prime} \mathrm{W}$, Sandoval-Ortega 736 (HUAA); $600 \mathrm{~m}$ al

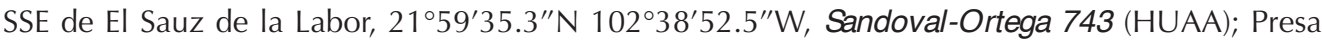

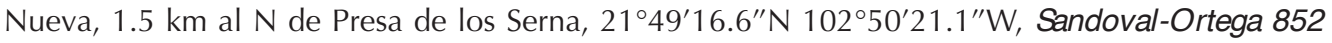
(HUAA); Exposición S de la cañada Los Adobes, $21^{\circ} 48^{\prime} 32^{\prime \prime} \mathrm{N} 102^{\circ} 42^{\prime} 10^{\prime \prime} \mathrm{W}$, De la Cerda-Lemus 6675 (HUAA; IEB); Cerro la Iguana, $4.5 \mathrm{~km}$ al NNW de El Sauz, $21^{\circ} 54^{\prime} 56.6^{\prime \prime} \mathrm{N} 102^{\circ} 38^{\prime} 36.8^{\prime \prime} \mathrm{W}$, Sandoval-Ortega 904 (HUAA). El Llano: Rancho Los Robles, $1.5 \mathrm{~km}$ al NE de Ojo de Agua de Crucitas, $21^{\circ} 58^{\prime} 04.1^{\prime \prime} \mathrm{N} \mathrm{101}{ }^{\circ} 56^{\prime} 12.1^{\prime \prime} \mathrm{W}$, Sandoval-Ortega 673 (HUAA); $1.7 \mathrm{~km}$ al NE de El Tule, $21^{\circ} 54^{\prime} 45.4^{\prime \prime} \mathrm{N} 101^{\circ} 55^{\prime} 06.4^{\prime \prime} \mathrm{W}$, Sandoval-Ortega 435 (HUAA). Jesús María: 6 km al SSE de Tapias Viejas, $21^{\circ} 48^{\prime} 34.1^{\prime \prime} \mathrm{N} 102^{\circ} 31^{\prime} 26.4^{\prime \prime} \mathrm{W}$, Sandoval-Ortega 468 (HUAA); $3.4 \mathrm{~km}$ al NE de Gracias a Dios, $21^{\circ} 57^{\prime} 34.8^{\prime \prime} \mathrm{N} 102^{\circ} 29^{\prime} 44.7^{\prime \prime} \mathrm{W}$, Sandoval-Ortega 777 (HUAA). Pabellón de Arteaga: $1 \mathrm{~km}$ al W de Santiago, $22^{\circ} 07^{\prime} 00.4^{\prime \prime} \mathrm{N} 102^{\circ} 20^{\prime} 52.9^{\prime \prime} \mathrm{W}$, Sandoval-Ortega 613 (HUAA); Faldas del Cerro del Chiquihuite, Rosales-Carrillo 202 (HUAA); Falda SE de Cerro Alto, a 4.6 km al NW deEl Garabato, $22^{\circ} 05^{\prime} 27.0^{\prime \prime} \mathrm{N} 102^{\circ} 23^{\prime} 08.0^{\prime \prime} \mathrm{W}$, Sandoval-Ortega 882 (HUAA). Rincón de Romos: $11.75 \mathrm{~km}$ al NW de Rincón de Romos, $22^{\circ} 08^{\prime} 49.8^{\prime \prime} \mathrm{N} 102^{\circ} 21^{\prime} 52.9^{\prime \prime} \mathrm{W}$, Sandoval-Ortega 302 (HUAA). San Francisco

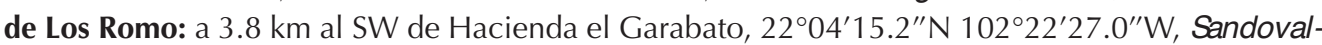
Ortega 839 (HUAA). San José de Gracia: Extremo NE de San Antonio de los Ríos, 22 ${ }^{\circ} 10^{\prime} 04.7^{\prime \prime} \mathrm{N}$ $102^{\circ} 28^{\prime} 03.0^{\prime \prime} \mathrm{W}$, Sandoval-Ortega 598 (HUAA). Tepezalá: Ladera N del cerro Mesillas, $22^{\circ} 19^{\prime} 54^{\prime \prime} \mathrm{N}$ $102^{\circ} 09^{\prime} 23^{\prime \prime}$ W, Martínez-Ramírez 1185 (HUAA; IEB).

\section{Salsola tragus $\mathrm{L}$.}

Aguascalientes: Rancho el Refugio, $21^{\circ} 47^{\prime} 37.3^{\prime \prime} \mathrm{N} 102^{\circ} 15^{\prime} 10.2^{\prime \prime} \mathrm{W}$, Sandoval-Ortega 868 (HUAA); Carrtera Aguascalientes-Jesús María, García-Regalado 4554 (HUAA); 1.5 km al E de Ags. (Ojo de Palmitas), De la Cerda-Lemus 5861 (HUAA); Ciudad Universitaria, UAA, Esparza S. 87 (HUAA); Al W de Cieneguilla, Lado E del Río San Pedro, Rosales-Carrillo 1926 (HUAA); Rumbo al Cerro de Los Gallos, Rosales-Carrillo 1968 (HUAA) Calvillo: 2 km al NE de Malpaso, Sandoval-Ortega 570 (HUAA); $3 \mathrm{~km}$ al SSW de La Labor, 22 ${ }^{\circ} 56^{\prime} 20.3^{\prime \prime} \mathrm{N} 102^{\circ} 42^{\prime} 48.1^{\prime \prime} \mathrm{W}$, Sandoval-Ortega 644 (HUAA). Cosio: Tanque La Punta, al E del Poblado La punta, Rosales-Carrillo 2059 (HUAA); 3 km al SSW de La Labor, $21^{\circ} 56^{\prime} 20.3^{\prime \prime} \mathrm{N} 102^{\circ} 42^{\prime} 48.1^{\prime \prime} \mathrm{W}$, Sandoval-Ortega 644 (HUAA); 2 km al NE de Mal-

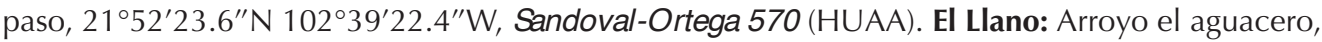
a $2.5 \mathrm{~km}$ al NE de El Tule, 21 ${ }^{\circ} 54^{\prime} 50.0^{\prime \prime} \mathrm{N} 101^{\circ} 54^{\prime} 25.7^{\prime \prime} \mathrm{W}$, Sandoval-Ortega 447 (HUAA); Rancho

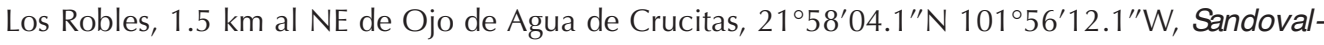

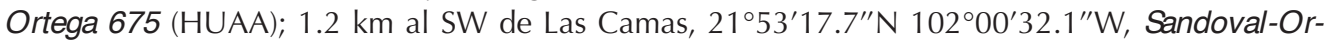
tega 614 (HUAA). Jesús María: $5 \mathrm{~km}$ al SE de Tapias Viejas (Cañada Guijolotes), 214ㅇ'54.1" $\mathrm{N}$ $102^{\circ} 31^{\prime} 30.4^{\prime \prime} \mathrm{W}$, Sandoval-Ortega 461 (HUAA); Carretera a Zacatecas, hacia el N de La Escalera, $22^{\circ} 01^{\prime} 45.4^{\prime \prime} \mathrm{N} 102^{\circ} 16^{\prime} 59.8^{\prime \prime} \mathrm{W}$, Arroyo-Chávez 273 (HUAA). Pabellón de Arteaga: Carretera a Pabellón de Arteaga, Rosales-Carrillo 1833 (HUAA). Rincón de Romos: Torre de Microondas de Rincón, Squeiros-Delgado 2187 (HUAA). San Francisco de Los Romo: $2 \mathrm{~km}$ al SE de San Fco. De Los Romo, 22 $2^{\circ} 03^{\prime} 14.9^{\prime \prime} \mathrm{N} 102^{\circ} 14^{\prime} 44.4^{\prime \prime} \mathrm{W}$, Sandoval-Ortega 676 (HUAA). Tepezalá: $500 \mathrm{~m}$ al E de Puerto de La Concepción, 22 $2^{\circ} 12^{\prime} 10.1^{\prime \prime} \mathrm{N} 102^{\circ} 07^{\prime} 52.4^{\prime \prime} \mathrm{W}$, Sandoval-Ortega 631 (HUAA). 